\title{
Chemical nonequilibrium for interacting bosons: Applications to the pion gas
}

\author{
D. Fernández-Fraile* and A. Gómez Nicola ${ }^{\dagger}$ \\ Departamento de Física Teórica II, Universidad Complutense, 28040 Madrid, Spain
}

(Received 9 March 2009; published 16 September 2009)

\begin{abstract}
We consider an interacting pion gas in a stage of the system evolution where thermal but not chemical equilibrium has been reached, i.e., for temperatures between thermal and chemical freeze-out $T_{\text {ther }}<$ $T<T_{\text {chem }}$ reached in relativistic heavy-ion collisions. Approximate particle number conservation is implemented by a nonvanishing pion number chemical potential $\mu_{\pi}$ within a diagrammatic thermal fieldtheory approach, valid in principle for any bosonic field theory in this regime. The resulting Feynman rules are derived here and applied within the context of chiral perturbation theory to discuss thermodynamical quantities of interest for the pion gas such as the free energy, the quark condensate, and thermal self-energy. In particular, we derive the $\mu_{\pi} \neq 0$ generalization of Luscher and Gell-Mann-OakesRenner-type relations. We pay special attention to the comparison with the conventional kinetic theory approach in the dilute regime, which allows for a check of consistency of our approach. Several phenomenological applications are discussed, concerning chiral symmetry restoration, freeze-out conditions, and Bose-Einstein pion condensation.
\end{abstract}

DOI: 10.1103/PhysRevD.80.056003

PACS numbers: 11.10.Wx, 12.39.Fe, 25.75.- $\mathrm{q}$

\section{INTRODUCTION}

One of the ongoing research lines in heavy-ion physics is the thermal and chemical evolution of the expanding hadronic gas. Roughly speaking, the accepted picture is that the evolution of the cooling system reaches chemical freeze-out before the thermal one, so that when hadrons fully decouple the chemical potentials associated with particle number conservation are not zero. The chemical composition of the gas can be determined experimentally by looking at the relative abundances of the different hadron species and their spectra [1-5]. The presence of such a chemically not-equilibrated phase is more likely to exist for higher collision energies such as those in the Relativistic Heavy Ion Collider (RHIC) or LHC than for Super Proton Synchrotron (SPS) or Alternating Gradient Synchrotron (AGS) experiments [4]. For the pion component, different estimates based on local thermal equilibrium and particle spectra analyses predict $\mu_{\pi} \sim$ 50-100 MeV at a thermal freeze-out temperature $T_{\text {ther }} \sim$ 100-120 MeV, with chemical freeze-out taking place at about $T_{\text {chem }} \sim 180 \mathrm{MeV}[2,3,5-8]$. On the other hand, the plasma is almost electrically neutral, so that it is a good approximation to keep vanishing charge or isospin chemical pion chemical potentials.

For low and moderate temperatures, the dominant component is the pionic one. In that phase, the mean-free path of pions is small compared to the system size, so that local thermal equilibrium prevails $[2,9,10]$. On the other hand, the chemical relaxation rate through inelastic $\pi \pi \leftrightarrows \pi \pi \pi \pi$ processes is very small $[8,11]$ due to a

\footnotetext{
*danfer@fis.ucm.es

†'gomez@fis.ucm.es
}

strong phase space suppression. Therefore, in the range of temperatures $T_{\text {ther }}<T<T_{\text {chem }} \lesssim T_{c}$, with $T_{c}$ the chiral restoration critical temperature, the system is in thermal equilibrium and dominated by elastic collisions so that $\mu_{\pi} \neq 0$. In that temperature range, it is valid to use the theoretical framework of chiral perturbation theory (ChPT) and it is also reasonable to adopt a dilute gas description, since the mean particle density is small. In addition, by neglecting dissipative effects such as viscosities, entropy is conserved in the evolution.

The system described above, i.e., a pion gas with $\mu_{\pi} \neq$ 0 is the one we will consider here. Clearly, it is an oversimplified version of the real hadron gas, but we will take it as a physically relevant working example for our present analysis. So far, pion number chemical potential effects in such a system have been incorporated basically in two ways. One of them is the limit of free particles (where one has actually exact particle conservation) used for the evaluation of the partition function, including resonances explicitly $[2,8]$. This allows, via entropy conservation requirements, to describe rather accurately the isentropic dependence $\mu_{\pi}(T)$ in the range of temperatures of phenomenological relevance indicated above. The other one is to use kinetic theory arguments to include the $\mu_{\pi} \neq 0$ dependence directly in the distribution function. The latter has been followed for instance in the calculation of the thermal width [9], in the evaluation of transport coefficients $[12,13]$, or in the virial approach for low densities [14]. Finally, it is worth mentioning that there are phenomenological analyses, similar to those in [15] for the dilepton rate, where the same prescription is followed, i.e., replacing the distribution function, but for propagators at the diagrammatic level, inspired on the nonequilibrium formulation of thermal field theory [16]. 
We are interested in a diagrammatic formulation of this system, i.e., we will derive the Feynman rules to be used when approximate particle number conservation is valid. The Feynman rules of thermal field theory with exact conserved charges can be obtained straightforwardly [17] but this is a completely different situation, since particle number is not exactly conserved in an interacting bosonic field theory (conserved only in the free case), and therefore there is not a local number operator to be added to the Lagrangian in the usual way. This will also be related to the impossibility to define a proper Matsubara imaginary-time formalism (ITF). The motivation for our field-theory description is twofold: first, it will provide a formal proof of the consistency and validity of the different prescriptions used in the literature and mentioned in the previous paragraph. Second, it will allow one to deal in a natural way with pion interactions when $\mu_{\pi} \neq 0$, which is particularly interesting in order to describe corrections to dynamical quantities such as the thermal pion self-energy, but also to evaluate the effect of interactions in thermodynamical observables.

The paper is organized as follows: in the first part we will describe our formalism, based on holomorphic path integrals, which naturally leads to the relevant Feynman rules. Although the results in that part are actually valid for any real scalar field theory provided one neglects the contributions of number-changing scattering processes or, in other words, if the gas is dilute enough, we will be primarily interested in the pion gas, where chemical nonequilibrium is actually reached during the expansion. We explain more clearly this physical motivation in Sec. II, where we discuss the relevant distribution function to describe the system. The Feynman rules we derive (Sec. III) had not been considered before in the interacting case and, as we will see, they are really meaningful only in the real-time formalism (RTF) of thermal field theory. The second part (Sec. IV) deals with the application of our formalism to the pion gas. We will analyze corrections both in thermodynamical (free energy, entropy, particle number, and quark condensate) and dynamical (thermal mass and width) observables, the former being understood as a generalization of the usual thermodynamical variables during the chemical nonequilibrium phase. We also compare to previous works in the literature and we discuss several phenomenological consequences regarding chiral symmetry restoration, Bose-Einstein condensation of neutral and charged pions, as well as thermal and chemical freeze-out. Appendixes A and B contain detailed results used in the main text about holomorphic path integrals and thermal propagators, respectively. In particular, in Appendix B we discuss some relevant differences between the case of particle number chemical potential considered here and the more usual one associated with the electric charge exact conservation, concerning especially the way in which Kubo-Martin-Schwinger (KMS) boundary conditions are broken.

\section{PHYSICAL MOTIVATION}

As stated in the Introduction, we are interested in describing the physical system constituted by a pion gas in expansion, during the time when the number of pions is approximately conserved. This is the case of the pionic component of the hadronic gas produced after a relativistic heavy-ion collision. The pionic component is the dominant one in the hadron gas around thermal freeze out [1], although considering additional degrees of freedom in the gas (kaons, etas, nucleons, and resonances) and the interactions among them is relevant at temperatures close to the chiral phase transition. We will not consider those extra components here, although their inclusion in the chiral framework, together with the corresponding additional chemical potentials (strangeness and baryon number) is a feasible extension of this work. Unlike other treatments [2], where it was shown that it is a reasonable approximation to introduce in the partition function all the states (asymptotic states as well as resonances) up to a given energy as free degrees of freedom, in our approach the resonances present in the pion gas, the $f_{0}(600) / \sigma$ and the $\rho(770)$, are generated dynamically by means of unitarization methods so the actual degrees of freedom in the Lagrangian are only pions. It is however important to mention that, even when introduced as explicit degrees of freedom, the processes $\rho \leftrightarrows \pi \pi$ and $\sigma \leftrightarrows \pi \pi$ do not restore chemical equilibrium in the pionic component, because $\mu_{\rho}=\mu_{\sigma}=2 \mu_{\pi}$, the truly relevant particlechanging process being $\pi \pi \leftrightarrows \pi \pi \pi \pi$. When pions and resonances are in chemical equilibrium with respect to each other we talk about a relative chemical equilibrium, since it is possible to choose their chemical potentials to maintain it, whereas absolute chemical equilibrium is only possible for $\mu_{\pi}=0$ [8].

The evolution of the pionic fireball can be divided into three stages as it cools down according to the corresponding temperature ranges [2]: (I) $T_{\text {chem }} \leq T \leq T_{c}$, the pion gas is produced after hadronization from a quark-gluon plasma phase and it is in full statistical equilibrium (thermal and chemical). (II) $T_{\text {ther }} \leq T \leq T_{\text {chem }}$, the mean-free path of elastic collisions, $\lambda_{\text {el }}$ is smaller than the typical size of the fireball, $R \sim 5-10 \mathrm{fm}$ [18], so that thermal equilibrium is maintained, whereas the mean-free path of inelastic collisions $\lambda_{\text {in }}$ is larger than $R$ so the total number of pions $N_{\pi} \equiv N_{\pi^{0}}+N_{\pi^{+}}+N_{\pi^{-}}$remains approximately constant $^{1}$ and a finite chemical potential associated to $N_{\pi}$ builds up, so that the system is out of chemical equilibrium. (III) $T \leq T_{\text {ther }}, \lambda_{\text {el }}$ is larger than $R$, and so the pions stop

\footnotetext{
${ }^{1}$ For instance, at $T=150 \mathrm{MeV}$ the relaxation time of elastic $\pi \pi$ collision is $\tau_{\mathrm{el}} \sim 2 \mathrm{fm}$, whereas the relaxation time of the process $\pi \pi \leftrightarrows \pi \pi \pi \pi$ is $\tau_{\text {in }} \sim 200 \mathrm{fm}$ [8], the typical mean velocities at those temperatures being $\bar{v} \sim c$. See also our comments in Sec. IV C.
} 
interacting and their momentum distributions become frozen, so that thermal equilibrium is lost.

We shall analyze phase II of the evolution, where the total number of pions is approximately conserved (and the gas remains dilute enough) so that the introduction of a finite pion chemical potential $\mu_{\pi}$ associated to $N_{\pi}$ is necessary. The existence of such a chemically notequilibrated phase during the fireball space-time evolution is supported by several phenomenological results. For instance, when analyzing experimental data from the NA44 Collaboration, it was shown in [3] that in order to properly fit the pion spectrum at low transverse momentum in $\mathrm{PbPb}$ reactions, one needs to introduce a finite chemical potential of order $\mu_{\pi} \sim 60-80 \mathrm{MeV}$ at thermal freeze-out. A similar conclusion is reached in [5] for RHIC AuAu collisions. In addition, the analysis of total particle yields and yield ratios for SPS and RHIC energies are fitted with values of fugacities compatible with the pionic component being significatively out of chemical equilibrium [6,7].

Neglecting electromagnetic interactions, the pions are described by neutral scalar fields. For a neutral boson field theory, the particle number is conserved only in the free case. Our aim here is to provide a field-theory description of the nonequilibrium state corresponding to phase II. In this respect, it is important to remark that there are fundamental differences between total particle number and charges which are exactly conserved by the dynamics, such as the net electric charge or baryon number in QCD. In a pion gas, the total number of pions is expressed in terms of individual number operators as $\hat{N}=$ $\hat{N}_{\pi^{0}}+\hat{N}_{\pi^{+}}+\hat{N}_{\pi^{-}}$, whereas electric charge, or the third isospin component [they are equivalent in the pion $S U(2)$ case] is measured by $N_{\pi^{+}}-N_{\pi^{-}}[19,20]$. The main difference is of course that charge is exactly conserved in the second case, which implies several important consequences: first, from the field-theoretical point of view, in the charge case there is a local charge operator $\hat{Q}$ written in terms of the field and its derivatives, which allows for a straightforward derivation of the corresponding Feynman rules, adding the usual $\mu_{Q} \hat{Q}$ term to the Lagrangian $[17,21]$. However, that is not the case for the particle number, which instead has a natural formulation in terms of canonical creation and annihilation operators. That is the main reason why we will develop our holomorphic representation of the path integral in Sec. III. Second, in the $\mu_{\pi}$ case we are really facing a nonequilibrium description, which is only consistent if $\mu_{\pi}$ and $T$ are not independent parameters, the function $\mu_{\pi}(T)$ parametrizing the deviations from chemical equilibrium and vanishing at $T=T_{\text {chem }}$. This signals the end of phase II, or its beginning if we think in terms of proper time, as inverse of temperature evolution in a hydrodynamical description (remember that in phase II local thermal equilibrium is assumed). The form of $\mu_{\pi}(T)$ has to be fixed by additional physical assumptions. We will rely here (see Sec. IV B) on the isentropic condition stating that the ratio of entropy density to pion density $s / n$ remains constant along the chemical evolution, which has phenomenological support $[2,3]$. Finally, we remark that these differences between the charge $\mu_{Q} \neq 0$ case and the pion number $\mu_{\pi} \neq 0$ one translate into a different way in which the KMS boundary conditions characteristic of equilibrium are broken. We discuss this issue in detail in Appendix B. Throughout this work we will take $\mu_{Q}=0$, which corresponds to an electrically neutral pion gas, which seems to be well supported by the phenomenological values of the fugacities [7].

Since the system in phase II is in thermal equilibrium and there is an approximate conserved operator $\hat{N}$ with chemical potential $\mu_{\pi}$ associated, the appropriate nonequilibrium partition function is

$$
\tilde{Z}_{\beta}(t) \equiv \operatorname{Tr}\left\{e^{-\beta\left(\hat{H}-\mu_{\pi} \hat{N}\right)}\right\},
$$

where quantities with a tilde will refer always to the nonequilibrium $\mu_{\pi} \neq 0$ case throughout this paper. By including source terms we can then derive thermal correlation functions. Note that $\tilde{Z}_{\beta}$ is independent of the position in space (we consider an homogeneous system), but it actually depends on (proper) time $t$ during the gas expansion through temperature $\beta(t) \equiv 1 / T(t)$ and the chemical potential $\mu(T(t))$, along the lines discussed in the previous paragraph. The validity of the out-of-(chemical) equilibrium distribution function in (1) will be subject to times $t<$ $t_{\mathrm{II}}$, where $t_{\mathrm{II}}$ is the duration of phase II. It is in this nonequilibrium effectively time-dependent situation that our results for the partition function and thermodynamical observables (see Sec. IVA) have to be understood.

For $t<t_{\mathrm{II}}$ inelastic processes are scarce at temperatures $T_{\text {ther }} \leq T \leq T_{\text {chem }}$, therefore if at some time $t_{1}$ the system is in a state with a well-defined number of particles equal to $N, \hat{N}\left|n\left(t_{1}\right)\right\rangle=N\left|n\left(t_{1}\right)\right\rangle$, then at another time $t_{2}$ with $t_{2}-$ $t_{1}\left\langle t_{\mathrm{II}}, \hat{N} \mid n\left(t_{2}\right)\right\rangle \simeq N\left|n\left(t_{2}\right)\right\rangle$, and thus in Heisenberg's picture $\hat{N}\left(t_{1}\right) \simeq \hat{N}\left(t_{2}\right)$ and from Heisenberg's equation $i d \hat{N}(t) / d t=[\hat{N}(t), \hat{H}]$ we infer

$$
\begin{aligned}
0 \simeq & i\left(\hat{N}\left(t_{2}\right)-\hat{N}\left(t_{1}\right)\right)=\int_{t_{1}}^{t_{2}}[\hat{N}(t), \hat{H}] d t \Rightarrow[\hat{N}(t), \hat{H}] \simeq 0 \\
& \text { for } t_{1} \leq t \leq t_{\mathrm{II}} .
\end{aligned}
$$

In the following section, the condition $[\hat{N}(t), \hat{H}] \simeq 0$ (valid for times in phase II) will be used to derive a field-theory description of the system based on the holomorphic pathintegral representation of the partition function provided by (1).

\section{FORMALISM: CHEMICAL POTENTIALS FOR NEUTRAL BOSONS}

As we mentioned in the Introduction, the operator $\hat{N}$ has a nonlocal representation in terms of the field operator 
[22], so that the appropriate representation for this operator is instead in terms of creation and annihilation operators. The holomorphic path-integral representation uses these convenient operators, and its main ideas can be found in [23]. We will give the essential steps of the derivation for our system in this section, with more technical aspects being relegated to Appendix A. As we saw in the previous section, the generating functional of thermal correlation functions will be constructed from the non-(chemical) equilibrium partition function (1). In order to further simplify the discussion, we will consider first a quantummechanical gas of Bose particles and next we will extend it straightforwardly to the quantum field theory (QFT) case. Throughout this section and for simplicity we use the notation $\mu$ for the chemical potential associated to the particle number ( $\mu_{\pi}$ in the pion gas case, which we will analyze extensively in Sec. IV).

Let us consider then a single-frequency quantum oscillator (free Hamiltonian) coupled to an external force $j(t)$. The Hamiltonian and number operators are then

$$
\begin{gathered}
\hat{H}=\frac{1}{2} \hat{p}^{2}+\frac{1}{2} \omega^{2} \hat{q}^{2}-j(t) \hat{q} \equiv \hat{H}_{0}-j(t) \hat{q} \\
=\frac{\omega}{2}\left(\hat{a}^{\dagger} \hat{a}+\hat{a} \hat{a}^{\dagger}\right)-\frac{1}{\sqrt{2 \omega}}\left(\hat{a}^{\dagger}+\hat{a}\right) j(t), \\
\hat{N}=\hat{a}^{\dagger} \hat{a},
\end{gathered}
$$

where $\hat{q}$ and $\hat{p}$ are, respectively, the position and the conjugate momentum operators (whose role will be played by the field and its conjugate momentum) and the creation and annihilation operators are defined in the usual way:

$$
\hat{a}=\frac{i}{\sqrt{2 \omega}}(\hat{p}-i \omega \hat{q}), \quad \hat{a}^{\dagger}=-\frac{i}{\sqrt{2 \omega}}(\hat{p}+i \omega \hat{q}),
$$

satisfying canonical commutation relations $\left[\hat{a}, \hat{a}^{\dagger}\right]=\hat{1}$.

In the holomorphic representation [23], traces of operators are evaluated in the space of complex analytic functions of one complex variable $z$ and creation and annihilation operators act on this space as

$$
\hat{a}^{\dagger} \mapsto z, \quad \hat{a} \mapsto \frac{\partial}{\partial z} .
$$

We have included in Appendix A some of the technical details to perform the relevant calculations in this formalism. In particular, the partition function for any Hamiltonian $\hat{H}$ reads, from (A7),

$$
\tilde{Z}_{\beta}=\int \frac{d z d \bar{z}}{2 \pi i} e^{-\bar{z} z}\left\langle z\left|e^{-\beta(\hat{H}-\mu \hat{N})}\right| \bar{z}\right\rangle .
$$

Now, if the number operator is approximately conserved, then $[\hat{H}, \hat{N}] \simeq 0$ and Eq. (7) can be recast, by inserting the identity once, as

$$
\tilde{Z}_{\beta} \simeq \int \frac{d z d \bar{z}}{2 \pi i} e^{-\bar{z} z} \int \frac{d z^{\prime} d \bar{z}^{\prime}}{2 \pi i} e^{-\bar{z}^{\prime} z^{\prime}}\left\langle z\left|e^{\beta \mu \hat{N}}\right| \bar{z}^{\prime}\right\rangle\left\langle z^{\prime}\left|e^{-\beta \hat{H}}\right| \bar{z}\right\rangle .
$$

This is the key step of the derivation, since it contains our main approximation, which is equivalent to consider only up to two-particle states in the trace (1). Therefore, it is physically appropriate to describe a dilute regime where elastic collisions dominate and particle number is approximately conserved.

Now, the first matrix element in (8) can be calculated directly, using (A14) with $j=0, t_{f}=t_{i}-i \beta$, and $\omega=$ $-\mu$ :

$$
\left\langle z\left|e^{\beta \mu \hat{N}}\right| \bar{z}^{\prime}\right\rangle=\exp \left(z \bar{z}^{\prime} e^{\beta \mu}\right)
$$

so that, using (A4) we arrive at

$$
\tilde{Z}_{\beta}=\int \frac{d z d \bar{z}}{2 \pi i} e^{-\bar{z} z}\left\langle z e^{\beta \mu}\left|e^{-\beta \hat{H}}\right| \bar{z}\right\rangle .
$$

From this representation of the partition function we define the corresponding generating functional (in the quantum mechanics case):

$$
\tilde{Z}_{\beta}[j] \equiv \int \frac{d z d \bar{z}}{2 \pi i} e^{-\bar{z} z}\left\langle z e^{\beta \mu}\left|e^{-\beta(\hat{H}-j \hat{q})}\right| \bar{z}\right\rangle,
$$

so that correlators of any function of the position operator $\hat{q}$ (the field operator in the QFT case) can be expressed in terms of functional derivatives of $\tilde{Z}_{\beta}[j]$ with respect to $j$ at $j=0$ in the usual way.

We will now proceed to the evaluation of $\tilde{Z}_{\beta}[j]$ when the Hamiltonian is the free one plus the source term, i.e., $\hat{H}=$ $\hat{H}_{0}-j \hat{q}$ in (3). Then, as usual, by functional derivation we will get the generating functional for the interacting case. We first separate the normal-ordered part as customary, i.e., $\hat{H}_{0}=\omega / 2+\omega \hat{a}^{\dagger} \hat{a}$, where the first term is the vacuum energy. Therefore, we have

$$
\tilde{Z}_{\beta}^{0}[j]=e^{-\beta \omega / 2} \int \frac{d z d \bar{z}}{2 \pi i} e^{-\bar{z} z} \mathcal{U}_{0}\left(z e^{\beta \mu}, \bar{z} ;-i \beta\right) .
$$

The function $\mathcal{U}$ is defined in (A10) and for the present case, its expression is given in (A14) and (A15). For its evaluation, we have considered, as detailed in Appendix A, the complex time contour shown in Fig. 1 joining the points $t_{i}$ and $t_{i}-i \beta$ with $\sigma \in[0, \beta]$, which contains the usual real-time and imaginary-time paths of thermal field theory and satisfies the usual requirements for the path integral to be well defined. i.e., $\operatorname{Im} t$ is monotonically decreasing along the contour [17]. The imaginary-time contour runs in a straight line from $t_{i}=0$ down to $-i \beta$ and is denoted as $C_{4}$, while the $C_{1}$ and $C_{2}$ are the paths used in the real-time formulation (see below). 


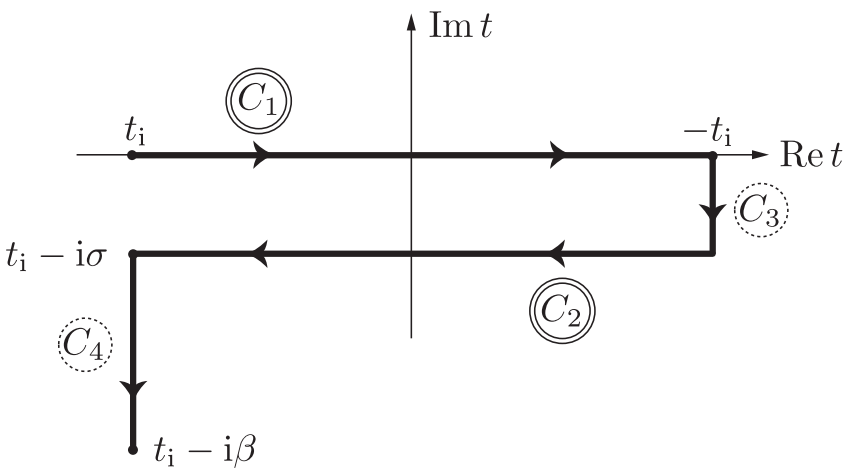

FIG. 1. Complex time contour including real- and imaginarytime paths, used in the derivation of the $\mu \neq 0$ Feynman rules, where $\sigma \in[0, \beta]$.

Now, replacing in (A14) and (A15) $z \rightarrow z e^{\beta \mu}, \bar{z}^{\prime} \rightarrow \bar{z}$, $t_{f} \rightarrow t_{i}-i \beta$ is equivalent to replace

$$
\beta \rightarrow \tilde{\beta}=\beta\left(1-\frac{\mu}{\omega}\right)
$$

except for the $\beta$ appearing in the $C$ contour. With this replacement one can follow the same steps as in [23] for the evaluation of the remaining integral in (12). Namely, one goes back to the discretized version of the path integral (see Appendix A), uses again (A13) with the modified $A$ matrix, and finally takes again the continuum limit. The final result is

$$
\tilde{Z}_{\beta}^{0}[j]=\tilde{Z}_{\beta}^{0} \exp \left(-\frac{1}{2} \int_{C} d t d t^{\prime} j(t) \tilde{G}_{\mathrm{F}}\left(t-t^{\prime}\right) j\left(t^{\prime}\right)\right),
$$

with the free partition function

$$
\tilde{Z}_{\beta}^{0}=\frac{e^{-\beta \omega / 2}}{1-e^{-\beta(\omega-\mu)}},
$$

and the free propagator:

$$
\tilde{G}(t)=\frac{1}{2 \omega}\left[e^{-i \omega|t|}(1+n(\omega-\mu))+e^{i \omega|t|} n(\omega-\mu)\right]
$$

where the Bose-Einstein function:

$$
n(x)=\frac{1}{e^{\beta x}-1},
$$

so that $n(\omega-\mu)$ is the free distribution function at nonzero $\mu$ in (B7) for a particle of positive energy. Note that we must restrict to $\mu<\omega$ in order that the previous expressions for the partition function are well defined (see also comments in Appendix B). The upper limit would correspond to Bose-Einstein condensation (see below). In the above propagator, $\left|t-t^{\prime}\right|$ has to be understood in terms of the relative position of times $t$ and $t^{\prime}$ with the path routing shown in Fig. 1.

The result (14) for the quantum-mechanical case for $\mu \neq 0$ is one of our main results. Its importance relies on the fact that we can now easily construct the generating functional in the interacting case, say $\hat{H}=\hat{H}_{0}+V(\hat{q})$ with $V$ the potential, in the usual way, i.e., by expanding formally in series of $V$ and writing every term in the expansion in terms of functional derivatives of $\tilde{Z}_{\beta}^{0}[j]$ with respect to $j$. From there, the extension to a QFT for a real scalar field $^{2}$ with Lagrangian density:

$$
\mathcal{L}=\frac{1}{2}\left(\partial_{\mu} \phi\right)^{2}-\frac{m^{2}}{2} \phi^{2}-\mathcal{V}(\phi)-j \phi
$$

is given by

$$
\begin{aligned}
\tilde{Z}_{\beta}[j]= & \tilde{Z}_{\beta}^{0} \exp \left(-i \int_{C} d^{4} x \mathcal{V}\left(\frac{\delta}{i \delta j(x)}\right)\right) \\
& \times \exp \left(-\frac{1}{2} \int_{C} d^{4} x \int_{C} d^{4} x^{\prime} j(x) \tilde{G}\left(x-x^{\prime}\right) j\left(x^{\prime}\right)\right),
\end{aligned}
$$

where $\int_{C} d^{4} x \equiv \int_{C} d t \int d^{3} \vec{x}$.

The generating functional (19) for the interacting case at $\mu \neq 0$ and the corresponding Feynman rules which we discuss below constitute central results of this paper and, to the best of our knowledge, they had not been considered before. It is valid for any scalar theory, provided one works in the regime where elastic collisions dominate and particle number is approximately conserved. The propagator $\tilde{G}$ appearing in (19) is the generalization of (16) to the QFT case when $\omega \rightarrow E_{p}$, the particle energy, and therefore we will be restricted in the following to $\mu \leq m$. Recall that the QFT generalization of $\tilde{\beta}$ in (13) is $\tilde{\beta}_{p}$ in (B13). The explicit expression of the propagator coincides, as it should, with the free two-point functions (B8)-(B11) derived in Appendix B directly within the canonical formalism at $\mu \neq 0$ for $t \in \mathbb{R}$. In this sense, one could somehow expect that the generalization to $\mu \neq 0$ of the generating functional is the one given in (19), although there was no rigorous proof available in the literature. We insist that the usual field-theory derivation for the case of an exactly conserved charge is not applicable here.

Next, we will discuss the Feynman rules needed for diagrammatic calculations. The $\mu \neq 0$ case for approximate particle conservation is essentially a nonequilibrium situation, as commented on several times before and thus it presents many subtleties to bear in mind. One of them is the impossibility of defining properly a Matsubara or imaginary-time formalism, which is related to the way in which the KMS conditions are broken. We will separate this discussion from the real-time case, where a suitable

\footnotetext{
${ }^{2} \mathrm{We}$ remark that in the field-theory case, whereas the Hamiltonian can be expressed as a space integral of a local field operator, that is not the case for the number operator when infinite frequencies appear. This is only possible for exactly conserved currents.
} 
formulation is possible, at least to the order we are considering here.

\section{A. Imaginary-time formalism}

The ITF corresponds to the choice $t_{i}=\sigma=0$ for the contour in Fig. 1 so that one is left only with purely imaginary times $t=-i \tau$ with $\tau \in[0, \beta]$. At $\mu=0$, this formalism is usually best suited to deal with thermodynamic quantities such as the free energy, while retarded Green functions can be derived from it by analytic continuation in the external frequencies [24]. However, the $\mu \neq 0$ propagator shows a distinctive feature that complicates diagrammatic calculations, generating in some cases ill-defined results. The origin of the problem is the way in which the standard equilibrium KMS periodicity conditions are broken. As explained in Appendix B, to which we refer for notation, in our case (particle number conservation valid both for neutral and charged bosons) we have, in the mixed representation of the propagator, $\tilde{\Delta}_{T}(\tau+$ $\left.\tilde{\beta}_{p}, p\right)=\tilde{\Delta}_{T}(\tau, p)$ instead of the familiar KMS condition $\tilde{\Delta}_{T}(\tau+\beta, p)=\tilde{\Delta}_{T}(\tau, p)$. This momentum-dependent periodic condition makes it impossible to define properly a Matsubara representation in Fourier space, which can only be done for $\tau \in\left[-\tilde{\beta}_{p}, \tilde{\beta}_{p}\right]$, e.g., Eq. (B20), instead of the required $[-\beta, \beta]$ interval where time differences appearing in propagators are evaluated (note that $\tilde{\beta}_{p}<\beta$ ). As explained in Appendix $B$, this departure of the standard equilibrium KMS condition in the particle number case is crucially different from that of a chemical potential associated with an exact charge conservation, like the electric charge for charged particles. In the charge case, the departure of KMS is given by the constant $\mu_{Q}$-dependent multiplicative factor in (B27) and, as explained in the Appendix, there is no obstruction to define the Matsubara representation for $\tau \in[-\beta, \beta]$ in that case, e.g., Eq. (B28), which amounts just to a shift in the Matsubara frequencies.

Turning again to the case analyzed in this paper, the KMS breaking mentioned in the above paragraph may be a problem for instance in diagrams contributing to the partition function (closed diagrams) whenever there is momentum exchange (time propagation) inside the diagram, i.e., more than one interaction vertex, since in that case the imaginary-time variables running in the internal propagators lie in the interval $[-\beta, \beta]$, while those propagators are only $\beta_{p}$ periodic. When there is just one interaction vertex, time integration factorizes trivially and the answer is proportional to powers of the tadpole-like contribution $\tilde{\Delta}_{T}(\tau=0, \vec{x}=0)$ given in Appendix B. That will be the case for all the contributions to leading order $\mathcal{O}\left(T^{6}\right)$ in the calculation of the ChPT partition function. The diagrams that contribute are given in Fig. 2 (see Sec. IVA). However, consider, for instance, the diagram labeled $8 \mathrm{~b}$ in Fig. 2, contributing to the ChPT free-energy density to $\mathcal{O}\left(T^{8}\right)$. Taking for simplicity constant vertices, as in the case of

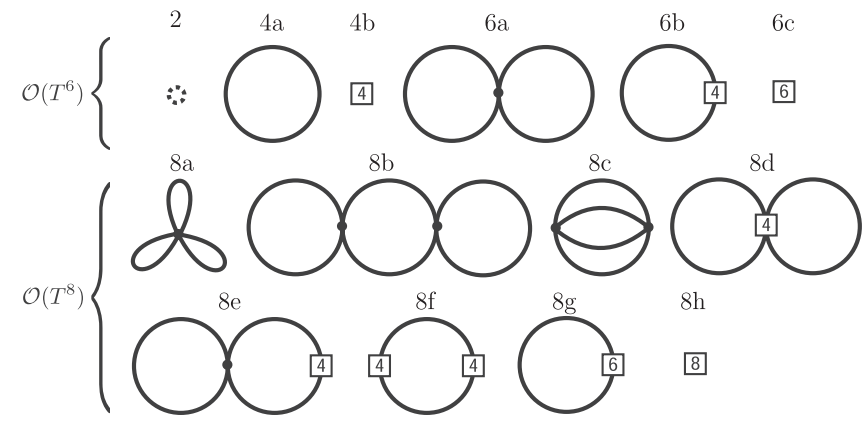

FIG. 2. Feynman diagrams contributing to the partition function of the pion gas up to and including $\mathcal{O}\left(T^{8}\right)$. The first row includes diagrams up to $\mathcal{O}\left(T^{6}\right)$, while the second and third rows are the $\mathcal{O}\left(T^{8}\right)$ contributions. The dots denote interaction vertices coming from $\mathcal{L}^{2}$, while those vertices coming from higher-order Lagrangians are indicated by a square box. The notation is the same as in [31].

$\mathcal{V}=\lambda \phi^{4} / 4$ !, this diagram in the ITF would be proportional to

$$
\begin{aligned}
I= & \frac{\tilde{G}^{2}(0)}{\beta} \int \frac{d^{3} \vec{p}}{(2 \pi)^{3}} \int_{0}^{\beta} d \tau^{\prime} \int_{0}^{\beta} d \tau \tilde{\Delta}_{T}\left(\tau-\tau^{\prime}, p\right) \\
& \times \tilde{\Delta}_{T}\left(\tau^{\prime}-\tau, p\right)
\end{aligned}
$$

with $\tilde{G}(0)=\tilde{\Delta}_{T}(0)$ given in Eqs. (B29)-(B31). Now, as commented above, we cannot just replace the Fourier representation for $\tilde{\Delta}_{T}$ in (B20) since it is only defined for the $\left[-\tilde{\beta}_{p}, \tilde{\beta}_{p}\right]$ interval. This obstruction produces additional unnatural terms. The appearance of those terms can be seen by using the mixed representation for $\Delta_{T}$ in terms of $\tilde{G}^{>}$and $\tilde{G}^{<}$given in (B10) and (B11) and performing explicitly the $\tau, \tau^{\prime}$ integrals in (20). We get

$$
\begin{aligned}
I= & -\tilde{G}^{2}(0) \frac{\partial}{\partial m^{2}} \tilde{G}(0)+\frac{\tilde{G}(0)}{\beta} \int \frac{d^{3} \vec{p}}{(2 \pi)^{3}} \frac{1}{8 E_{p}^{4}} \\
& \times\left\{\left[1+\tilde{n}_{p}\left(E_{p}\right)\right]^{2}\left[e^{2 \beta \mu}-1\right]+\left[\tilde{n}_{p}\left(E_{p}\right)\right]^{2}\left[e^{-2 \beta \mu}-1\right]\right\},
\end{aligned}
$$

with $E_{p}^{2}=|\vec{p}|^{2}+m^{2}$. The first term above gives the standard result for $\mu=0$ with the replacement of the distribution function $n \rightarrow \tilde{n}$, as one would expect from kinetic theory arguments, while this property does not hold for the additional terms. The remaining contributions vanish for $\mu=0$ but they do not do so in the $T \rightarrow 0^{+}$limit where they diverge. This contradicts the natural physical expectation that in the $T \rightarrow 0^{+}$limit and for $\mu<m$, the free energy should reduce to the vacuum contribution.

A related conflict arises when trying to calculate correlation functions in the ITF. The loss of KMS $\beta$ periodicity implies that the dependence on external times is not only through time differences. In particular, this means that correlators depend on $t_{i}$. Consider for instance the tadpole-like contribution (we omit the spatial dependence 
for simplicity):

$$
\begin{aligned}
& \int_{i t_{i}}^{i t_{i}+\beta} d \tau \tilde{\Delta}_{T}\left(\tau_{1}-\tau\right) \tilde{\Delta}_{T}\left(\tau-\tau_{2}\right) \\
& \quad=\int_{\tilde{T}}^{\tilde{T}+\beta} d \tau \tilde{\Delta}_{T}\left(\tau_{1}-\tau_{2}-\tau\right) \tilde{\Delta}_{T}(\tau),
\end{aligned}
$$

where $\tilde{T}=i t_{i}-\tau_{2}$. Now, if we make $\int_{\tilde{T}}^{\tilde{T}+\beta}=$ $\int_{0}^{\beta}-\int_{0}^{\tilde{T}}+\int_{\beta}^{\tilde{T}+\beta}$, the change of variable $\tau \rightarrow \tau+\beta$ in the third integral does not cancel the second one due to the loss of $\beta$ periodicity of $\tilde{\Delta}_{T}$. Therefore, the result does not depend only on $\tau_{1}-\tau_{2}$ but on $\tilde{T}$, i.e., depends on $\tau_{1}$ and $\tau_{2}$ independently and, as a consequence, the dependence on $t_{i}$ does not vanish.

As we will see in Secs. IVA and IV C, terms of the type shown above appear in the self-energy to leading order and in the partition function at order $\mathcal{O}\left(T^{8}\right)$. In the latter case, our approximation reaches its validity limit, since particlechanging processes start playing an important role. However, precisely for that reason, at the temperatures where the $\mathcal{O}\left(T^{8}\right)$ needs to be included we may consider in practice $\mu \ll T, m$ for these contributions. Recall that, in fact, the conflictive terms in $(21)$ are $\mathcal{O}(\mu / T)$ so that we will be introducing only small corrections by neglecting them. The presence of those unnatural terms in the ITF may also be understood if we note that we are facing a nonequilibrium situation, where the ITF is not appropriate and which must be formulated using a contour including real times $[16,25]$. We will indeed see next that one can define a suitable RTF so that these problems are not present, at least to the order we consider here, and one can calculate properly not only thermal correlators but also vacuum diagrams contributing to the free energy.

\section{B. Real-time formalism}

We consider now the full contour in Fig. 1 and, following the standard notation, we denote by $\tilde{D}_{i j}=\tilde{G}\left(t_{i}-t_{j}\right)$ with $t_{i} \in C_{i}, t_{j} \in C_{j}$. We then have for the $C_{1,2}$ parts of the contour (we omit the spatial dependence for simplicity):

$\tilde{D}_{11}\left(t-t^{\prime}\right)=\tilde{G}^{>}\left(t-t^{\prime}\right) \theta\left(t-t^{\prime}\right)+\tilde{G}^{<}\left(t-t^{\prime}\right) \theta\left(t^{\prime}-t\right)$,

$\tilde{D}_{22}\left(t-t^{\prime}\right)=\tilde{G}^{<}\left(t-t^{\prime}\right) \theta\left(t-t^{\prime}\right)+\tilde{G}^{>}\left(t-t^{\prime}\right) \theta\left(t^{\prime}-t\right)$,

$\tilde{D}_{12}\left(t-t^{\prime}\right)=\tilde{G}^{<}\left(t-t^{\prime}+i \sigma\right)=\tilde{D}_{21}\left(t^{\prime}-t\right)$,

where $t, t^{\prime} \in \mathbb{R}$ and $\tilde{G}^{>}, \tilde{G}^{<}$given in (B10) and (B11) and so on for the remaining components.

In order to formulate properly the RTF at $\mu \neq 0$ we take first, as customary, $t_{i} \rightarrow-\infty$. This is necessary if we want to calculate Green functions with arbitrary real-time arguments. In principle, this choice implies also that, imposing vanishing asymptotic conditions for the $j$ currents and for the spectral function, which hold also in our case, the generating functional for $\mathcal{V}=0$ can be factorized as [17]

$$
\tilde{Z}_{\beta, C}^{\mathcal{V}=0}[j]=\mathcal{N} \tilde{Z}_{\beta, C_{12}}^{\mathcal{V}=0}[j] \tilde{Z}_{\beta, C_{34}}^{\mathcal{V}=0}[j]
$$

so that one could calculate real-time correlation functions without worrying about the imaginary-leg contributions. However, as it was pointed out in [26,27], there are imaginary-time contributions that still survive in particular diagrams, for instance self-energy insertions, which indeed we will calculate here. Nevertheless, there is a standard rule for collecting all the relevant contributions but using only the propagators in $C_{1,2}$, the so-called $\left|p_{0}\right|$ prescription $[24,26,27]$. This prescription amounts to use in Fourier space $n\left(\left|p_{0}\right|\right)$ instead of the seemingly equivalent $n\left(E_{p}\right)$ when multiplied by the on-shell $\delta$ function, as in (B19). For instance, with this prescription one obtains that a simple constant tadpole-like insertion in the self-energy such as the diagram shown in Fig. 3(a) with a constant vertex, amounts to a redefinition of the mass, as expected. It also guarantees that there are no ill-defined contributions, such as products of $\delta$ distributions at the same point which in principle could appear when multiplying the RTF propagators. What we will show here is that for $\mu \neq 0$ there is also a natural prescription which works, now in terms of $n \rightarrow \tilde{n}_{p}\left(p_{0}\right)$, leading to the same properties at the order considered here. However, it must be pointed out that to higher orders, there may be additional ill-defined terms arising from a nonequilibrium distribution [25]. Our RTF avoids the main obstruction that we faced in the ITF, since the length $\beta$ of the imaginary leg disappears from the integration limits in momentum space, whose Fourier representation is well defined now. Moreover, we also choose $\sigma \rightarrow 0^{+}$. Therefore, for Green functions with real-time arguments for which we neglect (with the above prescription) the $C_{3,4}$ parts, we end up with a Keldysh-like contour characteristic of nonequilibrium thermal field theory [16]. With this procedure we will see that an additional property holds: most results can be written as functionals of $\tilde{n}$, which encodes all the $T, \mu$ dependence. This is also an expected property from kinetic theory arguments, at least for the leading-order corrections in $\tilde{n}$ (dilute gas regime).

This allows one then to calculate properly any real-time correlation function directly, i.e., without appealing to the analytic continuation from the ITF, which is cumbersome for $\mu \neq 0$. In addition, as we will see below, one can also
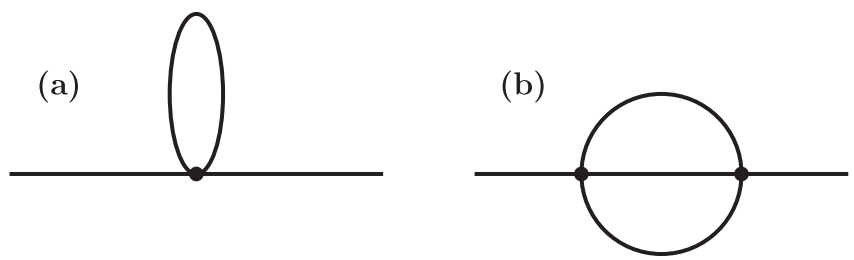

FIG. 3. Diagrams contributing to leading order to the real (a) and imaginary (b) parts of the self-energy. 
obtain information about the free-energy density without using the ITF. Let us then write the propagators (23) in momentum space for our choice of contour [note that the $\tilde{D}_{11}$ component corresponds to the free propagator $\tilde{G}$ in (B19)]:

$$
\begin{aligned}
& \tilde{D}_{11}\left(p_{0}, p\right)=\frac{i}{p_{0}^{2}-E_{p}^{2}+i \epsilon}+2 \pi \delta\left(p_{0}^{2}-E_{p}^{2}\right) n\left(\left|p_{0}\right|-\mu\right), \\
& \tilde{D}_{22}\left(p_{0}, p\right)=\frac{-i}{p_{0}^{2}-E_{p}^{2}+i \epsilon}+2 \pi \delta\left(p_{0}^{2}-E_{p}^{2}\right) n\left(\left|p_{0}\right|-\mu\right), \\
& \tilde{D}_{12}\left(p_{0}, p\right)=2 \pi \delta\left(p_{0}^{2}-E_{p}^{2}\right)\left[\theta\left(-p_{0}\right)+n\left(\left|p_{0}\right|-\mu\right)\right], \\
& \tilde{D}_{21}\left(p_{0}, p\right)=2 \pi \delta\left(p_{0}^{2}-E_{p}^{2}\right)\left[\theta\left(p_{0}\right)+n\left(\left|p_{0}\right|-\mu\right)\right] .
\end{aligned}
$$

In the above propagators, we have chosen, as discussed above, the $\left|p_{0}\right|$ prescription ensuring that the distribution function does not depend explicitly on $E_{p}$, as in the $\mu=0$ case. We will see below that this yields the same expected properties as for $\mu=0$. It can also be readily checked that our $\mu \neq 0$ RTF propagators above coincide with those given in [15], obtained assuming a direct replacement of the distribution function by the $\mu \neq 0$ nonequilibrium one. In fact, the free propagators (25) can be readily recast into the general nonequilibrium Keldysh form, given for instance in $[16,25]$ by taking for the nonequilibrium distribution function our $\tilde{n}_{p}\left(k_{0}\right)$ given in (B12) and (B13), ${ }^{3}$ which satisfies the property (B14), as required for general nonequilibrium derivations [25].

To provide a particularly relevant example of our previous statements, let us consider the tadpole-like correction to the self-energy given by the diagram in Fig. 3(a) with a constant vertex (the generalization to derivative vertices appearing in ChPT calculations will be straightforward). The external leg is fixed to be of "type 1," since we are calculating the two-point function with real arguments, i.e., the first-order correction to $D_{11}$. Then, if we consider only the $C_{1,2}$ contributions, this diagram gives in position space

$$
F(x-y)=i \sum_{j=1,2} \int_{C_{j}} \tilde{D}_{1 j}(x-z) \tilde{D}_{j j}(0) \tilde{D}_{j 1}(z-y) \text {. }
$$

Note that correlators depend only on space and time differences in the RTF, so that the problems discussed in the previous section, related to the ITF version of the tadpole in Eq. (22), are not present now.

Now, we take into account that $\tilde{D}_{11}(0)=\tilde{D}_{22}(0)=\tilde{G}(0)$ in (B29)-(B31). Then, the Fourier transform of $F$ is

$$
F\left(p_{0}, p\right)=i \tilde{G}(0)\left[\tilde{D}_{11}^{2}\left(p_{0}, p\right)-\tilde{D}_{12}\left(p_{0}, p\right) \tilde{D}_{21}\left(p_{0}, p\right)\right] .
$$

We replace in the above equation the propagators in (25) and use $\frac{\delta(x)}{x+i 0^{+}}=-\frac{\delta^{\prime}(x)}{2}-i \pi \delta^{2}(x)$, where as customary we

\footnotetext{
${ }^{3}$ The convention in [25] is such that the $D_{12}$ and $D_{21}$ components are reversed with respect to ours.
}

keep the regulator in the definition of $\delta(x)=\frac{i}{2 \pi}\left(\frac{1}{x+i 0^{+}}-\right.$ $\left.\frac{1}{x-i 0^{+}}\right)$. Thus, we can write

$$
\begin{aligned}
F\left(p_{0}, p\right)= & i \tilde{G}(0)\left\{\left[\frac{i}{p_{0}^{2}-E_{p}^{2}+i 0^{+}}\right]^{2}\right. \\
& \left.-2 \pi i n\left(\left|p_{0}\right|-\mu\right) \delta^{\prime}\left(p_{0}^{2}-E_{p}^{2}\right)\right\} \\
= & -\tilde{G}(0) \frac{\partial}{\partial m^{2}} \tilde{D}_{11}\left(p_{0}, p\right) .
\end{aligned}
$$

Note that it is in the last step in the previous equation where it is crucial to use the $\left|p_{0}\right|$ prescription chosen above since $n\left(\left|p_{0}\right|-\mu\right)$ is independent of $m^{2}$. Therefore, the result (28) implies that the only modification in the $\tilde{D}_{11}$ propagator is $m^{2} \rightarrow m^{2}-\tilde{G}(0)$, which is the expected result of mass renormalization which in addition is obtained from the $\mu=0$ case by replacing $n \rightarrow \tilde{n}$ in the (finite) thermal correction to the tadpole diagram given by the function $\tilde{g}_{1}(m, T, \mu)$ in (B31). Note that to this order and with this prescription we have been able to get rid of the ill-defined $\delta^{2}$ terms. However, this prescription might not be enough when higher orders are included, since it has been shown in [25] for a $\lambda \phi^{4}$ theory that additional nonequilibrium ill-defined terms arise, which should be properly regulated with a nonzero particle width. At the order corresponding to our previous result (28) we coincide with [25]. We will comment more about this issue in Sec. IV C.

Two more important remarks are in order. The first one is that the spectral properties of the interacting theory are really defined from retarded Green functions, not from time-ordered ones. From the ITF, retarded correlators are defined directly by analytic continuation. However, we have seen that this is not a well-defined procedure for $\mu \neq$ 0 . The solution of the problem of finding retarded Green functions from the RTF time-ordered product was given in [28]. In that work, a set of rules (the so-called circling rules) were provided in order to define a function that has the required causal retarded properties, namely, it satisfies that one of the outgoing lines of the corresponding diagram has the largest time component. It was then shown in several examples that this function coincides with the analytic continuation of the ITF correlator. Now, it can be checked that the same properties of the free propagators used in [28] for the derivation of the circling rules hold for our $\tilde{D}_{i j}$ propagators and therefore the same rules lead to the RTF retarded function at $\mu \neq 0$. The application of those rules is trivial for the tadpole case discussed above, since there is only one vertex. However, they will be of use for the case of higher-order contributions to the self-energy which we will consider below, like the thermal width arising from the diagram in Fig. 3(b).

The second remark has to do with the calculation of thermodynamic quantities within the RTF, i.e., the partition function or the free-energy density. In principle, due to the factorization of the imaginary-leg commented on above, 
the contribution to vacuum graphs when summing over fields of types 1 and 2 vanishes identically. However, it was shown in [29] that fixing one of the vertices of a vacuum diagram to be "external" of type 1 and summing over the remaining internal vertices with an overall $\beta$ factor reproduces the free-energy result and for $\mu=0$ coincides with the ITF. The functional arguments used in those papers are also applicable to our $\mu \neq 0$ case and, in fact, the direct use of that prescription leads to the expected answers. Let us show this for the case of the $\mathcal{O}\left(T^{8}\right)$ diagram 8 b in Fig. 2, analyzed in Sec. III A in the ITF. Applying the previous prescription and with constant vertices, we get the result that this diagram is now proportional to

$$
\begin{aligned}
& i \tilde{G}^{2}(0) \sum_{j=1,2} \int_{C_{j}} \tilde{D}_{1 j}(x-z) \tilde{D}_{j 1}(z-x)=\tilde{G}(0) F(0) \\
& =-\tilde{G}^{2}(0) \frac{\partial}{\partial m^{2}} \tilde{G}(0),
\end{aligned}
$$

with $F$ in (26). We then see that we arrive at the ITF result (21) but without the additional terms discussed in that section, since the proportionality factors between this diagram and (29) or (20) come only from combinatorics and are therefore identical. We will use this real-time prescription to properly define our free energy.

\section{APPLICATIONS TO THE PION GAS}

\section{A. Evaluation of the ChPT free energy}

We apply our previous results to the pion gas, described by ChPT with two light quark flavors of mass $m_{u}=m_{d} \equiv$ $m_{q}[30,31]$. The Lagrangian is constructed as an expansion in derivatives and pion masses, generically $\mathcal{O}(p)$ with $p \ll$ $\Lambda_{\chi} \sim 1 \mathrm{GeV}$, so that $\mathcal{L}=\mathcal{L}_{2}+\mathcal{L}_{4}+\cdots$ with $\mathcal{L}_{2 k}=$ $\mathcal{O}\left(p^{2 k}\right)$. In the range of temperatures and chemical potentials we are interested in, both $T, \mu_{\pi}=\mathcal{O}(p)$ formally, which corresponds to $T$ below $T_{c} \sim 200 \mathrm{MeV}$. The ChPT $\mathcal{O}\left(p^{D}\right)$ power of a given diagram is given by Weinberg's power counting $D=2\left(N_{L}+1\right)+\sum_{k} 2 N_{k}(k-1)$ [32], where $N_{L}$ is the number of loops and $N_{k}$ is the number of vertices coming from $\mathcal{L}_{2 k}$. In our approach, we do not perform any formal chiral expansion in $\mu_{\pi}$, except in higher-order contributions (see our discussion below and in Sec. III), where it is reasonable to expand in $\mu_{\pi} / T$. We will closely follow the notation and conventions in [31], where the explicit expressions of the $\mathcal{L}_{2}$ and $\mathcal{L}_{4}$ can be found. The Lagrangian $\mathcal{L}_{2}$ is the nonlinear- $\sigma$ model, whose free parameters are the pion decay constant and mass to leading order $f=f_{\pi}\left(1+\mathcal{O}\left(p^{2}\right)\right)$ with $f_{\pi} \simeq$ $93 \mathrm{MeV}$ and $m=m_{\pi}\left(1+\mathcal{O}\left(p^{2}\right)\right), m_{\pi} \simeq 140 \mathrm{MeV}$. To fourth order, $\mathcal{L}_{4}$ contains five independent low-energy constants $l_{1-4}$ and $h_{1}$ which absorb the divergences of the one-loop diagrams with only $\mathcal{L}_{2}$ vertices. The renormalized $\bar{l}_{i}$ appear in physical processes such as pion scattering and therefore their values can be fitted experimentally. We will use the same central values given in
[30,31] in order to compare more easily with the results in [31] at $\mu_{\pi}=0$. Those values are $\bar{l}_{1}=-6.6, \bar{l}_{2}=6.2$, $\bar{l}_{3}=2.9$, and $\bar{l}_{4}=3.5$. The constant $h_{1}$ multiplies a contact term and appears in the vacuum free energy and quark condensate. We use also the estimate in $[30,31]$ of $\bar{h}_{1} \simeq$ 3.4. The Lagrangians of higher orders will only appear through renormalization either of the vacuum energy or the pion mass and therefore the low-energy constants of those orders will not show up once the results are expressed in terms of the physical pion mass (see details below).

The free-energy density $z$, from which thermodynamical observables can be obtained, is defined as customary:

$$
\tilde{z}\left(T, \mu_{\pi}\right)=-T \lim _{V \rightarrow \infty} \frac{1}{V} \log \tilde{Z}_{\beta}\left(T, \mu_{\pi}\right) .
$$

We also define the thermodynamic pressure as in [31], i.e., subtracting its $T=0$ contribution given by the vacuum energy density:

$$
\tilde{P}\left(T, \mu_{\pi}\right)=\tilde{z}_{0}-\tilde{z}\left(T, \mu_{\pi}\right), \quad \tilde{z}_{0}=\lim _{T \rightarrow 0^{+}} \tilde{z} .
$$

It is important to emphasize that all of our results for the pressure and quantities derived from it have to be understood strictly as time dependent throughout the plasma expansion, in the sense explained in Sec. II, the time evolution toward a chemically equilibrated phase being driven by $\mu_{\pi}(T)$. The diagrams contributing to the free energy in ChPT are the closed diagrams shown in Fig. 2, where we follow the same convention as [31] to name the diagrams. The number assigned to each diagram indicates the order in the chiral expansion and the numbers inside the boxes in the vertices refer to the Lagrangian order, the case of $\mathcal{L}_{2}$ being indicated by a dot. Recall that for a given order of the Lagrangian, there are vertices with an arbitrary number of (even) pions due to the chiral expansion of the $S U(2)$-valued chiral field $U=\exp \left(i \pi^{a} \tau_{a} / f\right)$, where $\tau^{a}$ are the Pauli matrices and $\pi^{a}$ the pion field.

The leading order $\tilde{z}_{2}=-f^{2} m^{2}$, coming from the contact term (independent of the pion field) in $\mathcal{L}_{2}$, is independent of $T$ and $\mu_{\pi}$ and therefore contributes only to the vacuum energy density $\tilde{z}_{0}$. Note that, according to our discussion in the previous sections, we will ensure that all our contributions have a well-defined $T \rightarrow 0^{+}$limit for $\mu_{\pi}<m$, i.e., that the contributions to $\tilde{z}_{0}$ to any chiral order are $\mu_{\pi}$ independent. The next order corresponds to diagrams $4 \mathrm{a}$ and $4 \mathrm{~b}$ in Fig. 2. $\tilde{z}_{4 a}$ corresponds to the quadratic pion field contribution in $\mathcal{L}_{2}$ and is therefore nothing but the free partition function given in (B34) multiplied by 3 accounting for the 3 pion degrees of freedom. The divergent contribution to $\tilde{z}_{4 a}$ is $T$ and $\mu_{\pi}$ independent and therefore it merely renormalizes $\tilde{z}_{0}$.

The next order in the chiral expansion is $\mathcal{O}\left(T^{6}\right)$ and the diagrams contributing are $\tilde{z}_{6 a b c}$ in Fig. 2. It is important to remark that this is the first order where pion interactions show up. Graph $6 \mathrm{c}$ in Fig. 2 renormalizes $\tilde{z}_{0}$, while $6 \mathrm{~b}$ is of the same form as 4a in Fig. 2 and therefore gives rise to the 
free partition function contribution but with the mass shifted by its tree level $\mathcal{L}_{4}$ renormalization (see Sec. IV C) which depends on $l_{3}$. As for diagram 6a in Fig. 2, taking into account (B32), its contribution is proportional to $\tilde{G}^{2}(0)$. As discussed in Sec. III, in this case the result is trivially identical in both ITF and RTF and corresponds to the result in [31] replacing $G(0) \rightarrow \tilde{G}(0)$ :

$$
\tilde{z}_{6 a}=\frac{3 m^{2}}{8 f^{2}} \tilde{G}^{2}(0) .
$$

The divergent contribution in (32), according to (B29) and (B30), contains a contribution to $\tilde{z}_{0}$ and another one which cancels, as it should, with the one in $l_{3}$ so that, using (B35), the total finite result for the pressure to $\mathcal{O}\left(T^{6}\right)$ is

$$
\tilde{P}=\frac{3}{2} \tilde{g}_{0}\left(m_{\pi}, T, \mu_{\pi}\right)-\frac{3}{8} \frac{m^{2}}{f^{2}}\left[\tilde{g}_{1}\left(m, T, \mu_{\pi}\right)\right]^{2}+\mathcal{O}\left(T^{8}\right),
$$

with the functions $\tilde{g}_{1}$ and $\tilde{g}_{0}$ given in (B31) and (B33), respectively, and where $m_{\pi}$ is the physical pion mass at $T=\mu_{\pi}=0$, related to the bare mass $m$ to this order as [30]

$$
m_{\pi}^{2}=m^{2}\left[1-\frac{\bar{l}_{3}}{32 \pi^{2}} \frac{m^{2}}{f^{2}}+\mathcal{O}\left(m^{4}\right)\right] .
$$

Recall that, to this order, the difference between $m_{\pi}$ and $m$ is only relevant in the $\tilde{g}_{0}$ contribution in (33). The same applies to the distinction between $f$ and $f_{\pi}$ :

$$
f_{\pi}^{2}=f^{2}\left[1+\frac{\bar{l}_{4}}{8 \pi^{2}} \frac{m^{2}}{f^{2}}+\mathcal{O}\left(m^{4}\right)\right] .
$$

We consider now the $\mathcal{O}\left(T^{8}\right)$ contributions shown in Fig. 2. Now, there are several aspects which make the calculation qualitatively different from the $\mathcal{O}\left(T^{6}\right)$ one. An important point is that to this order we may expect that our approximation of particle number conservation is less accurate, since vertices entering number-changing processes show up. Consider for instance the diagrams contributing to $2 \pi \leftrightarrow 4 \pi$ processes in the thermal bath, which to leading order in ChPT are given by the tree-level diagrams shown in Figs. 4(a) and 4(b). Now, unlike the $\mathcal{O}\left(T^{6}\right)$ case, one can draw vacuum diagrams from these processes by identifying external lines. For instance, join- ing lines in pairs in the graph in Fig. 4(a) as 1-2, 3-4, 5-6 and equivalent combinations leads to diagram 8a in Fig. 2. Similarly, joining 1-2, 3-6, 4-5 in Fig. 4(b) produces diagram 8c. This is not a one-to-one correspondence. For instance, joining 1-3 and 2-4 lines in the elastic one-loop diagram in Fig. 4(c) yields also diagram 8c in Fig. 2. Diagram $8 \mathrm{~b}$ in Fig. 2 can also be obtained from an elastic process [Fig. 4(c) joining 1-2, 3-4] or from an inelastic one [Fig. 4(b) joining 1-3, 2-6, 4-5]. The crucial point is that none of the $\mathcal{O}\left(T^{6}\right)$ vacuum closed diagrams in Fig. 2 can be obtained from the lowest order inelastic diagrams in Figs. 4(a) and 4(b). This distinctive feature can be interpreted as a way to identify the validity range of our approximation. However, we should bear in mind that these $\mathcal{O}\left(T^{8}\right)$ corrections are meant to be relevant only very near $T_{c}$ [31] and therefore in the region where chemical equilibrium is nearly restored and $\mu_{\pi} \rightarrow 0$, not surprisingly due to the presence of the particle-changing processes just discussed [8]. Precisely for this reason, the $\mu_{\pi}$ dependence of these diagrams is suppressed in powers of $\mu_{\pi} / T$ and $\mu_{\pi} / m_{\pi}$. Therefore, numerically our approach will still be justified to this order. In addition, as we have explained in Sec. III, taking $\mu_{\pi} / T$ small justifies in practice to get rid of unnatural terms in the ITF formulation.

With the above considerations in mind, we proceed to evaluate the $\mathcal{O}\left(T^{8}\right)$ diagrams in Fig. 2. Graph $8 \mathrm{~h}$ renormalizes $\tilde{z}_{0}$ and graphs $8 \mathrm{f}$ and $8 \mathrm{~g}$ renormalize the pion mass to $\mathcal{O}\left(\mathrm{m}^{6}\right)$. Graph $8 \mathrm{a}$ is proportional to a third power of the propagator at the origin, with the same coefficient as in [31]:

$$
\tilde{z}_{8 a}=-\frac{25 m^{2}}{48 f^{4}} \tilde{G}^{3}(0)
$$

which contains divergent contributions, according to (B29).

The graph 8 b in Fig. 2 has been analyzed in Sec. III. The relevant integral contributing to this graph is (20) in the ITF and (29) in the RTF with the prescription discussed in that section. The difference between both formulations is of $\mathcal{O}\left(\mu_{\pi} / T\right)$ and therefore expected to be numerically small, for the reasons just discussed. The rest of the contributions to this graph are proportional to $\tilde{G}^{3}(0)$ and the proportionality constants are the same as in [31]. Thus, (a)

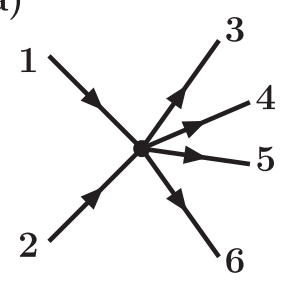

(b)

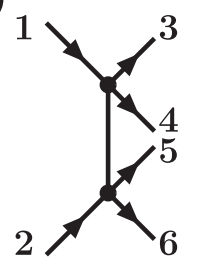

(c)

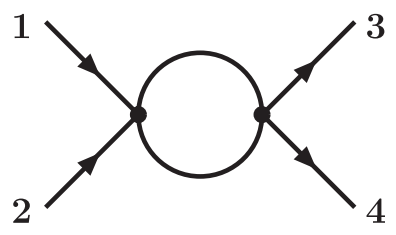

FIG. 4. (a), (b) Diagrams contributing to leading order (tree level) to $2 \pi \rightarrow 4 \pi$ processes. (c) A one-loop contribution to elastic $\pi \pi$ scattering. 
adopting the RTF prescription, we get

$$
\tilde{z}_{8 b}=\frac{m^{2}}{16 f^{4}} \tilde{G}^{2}(0)\left(8+3 m^{2} \frac{\partial}{\partial m^{2}}\right) \tilde{G}(0),
$$

whose divergent contribution can also be separated using (B29).

Graphs $8 \mathrm{~d}$ and $8 \mathrm{e}$ in Fig. 2 have the same form as graph 6a in (32), but due to the form of the $\mathcal{L}_{4}$ Lagrangian and following also our previous RTF prescriptions, we arrive at the same structure as in [31]:

$$
\begin{aligned}
\tilde{z}_{8 d}+\tilde{z}_{8 e}= & -\frac{3}{f^{4}}\left\{\left(2 l_{1}+4 l_{2}\right)\left[\tilde{G}_{\mu \nu}\right]^{2}\right. \\
& +\tilde{G}(0)\left[\left(3 l_{1}+l_{2}+l_{3}\right) m^{4} \tilde{G}(0)-\frac{l_{3}}{2} m^{6} \frac{\partial}{\partial m^{2}}\right] \\
& \times \tilde{G}(0)\},
\end{aligned}
$$

where $\tilde{G}_{\mu \nu}=\partial_{\mu} \partial_{\nu} \tilde{G}(0)$, which has the same properties as in [31], namely, its divergent contribution is the same ( $T$ and $\mu_{\pi}$ independent) while its finite part can be written in the same way in terms of $\tilde{g}_{0}$ and $\tilde{g}_{1}$.

The remaining graph is $8 \mathrm{c}$ in Fig. 2. Following again the RTF prescription, this contribution is

$$
\tilde{z}_{8 c}=\frac{1}{48 f^{4}}\left[3 m^{4} \tilde{J}_{1}-72 \tilde{J}_{2}+16 m^{2}(\tilde{G}(0))^{3}\right],
$$

where

$$
\begin{aligned}
\tilde{J}_{1}= & i \int d^{4} x\left[\tilde{D}_{11}^{4}(x)-\tilde{D}_{12}^{4}(x)\right] \\
= & \int d^{3} \vec{x} \int_{0}^{\infty} d t\left\{\left[\tilde{G}^{>}(t, \vec{x})\right]^{4}-\left[\tilde{G}^{<}(t, \vec{x})\right]^{4}\right\}, \\
\tilde{J}_{2}= & i \int d^{4} x\left[\left(\partial_{\mu} \tilde{D}_{11}(x) \partial^{\mu} \tilde{D}_{11}(x)\right)^{2}\right. \\
& \left.-\left(\partial_{\mu} \tilde{D}_{12}(x) \partial^{\mu} \tilde{D}_{12}(x)\right)^{2}\right] .
\end{aligned}
$$

Written in the above form, it is not difficult to show that for $\mu_{\pi}=0$, when the propagators are $\beta$ periodic, i.e., $G^{<}(t+i \beta)=G^{>}(t)$, one has for instance $J_{1}=$ $\int d^{3} \vec{x} \int_{0}^{\beta} d \tau \Delta_{T}^{4}(\tau, \vec{x})$ and similarly for $J_{2}$ [31]. As we have seen, for $\mu_{\pi} \neq 0$ the periodicity condition does not hold. However, for this diagram, instead of working directly with the RTF expressions (40), we will make use of the fact that $\tilde{G}^{<}(t+i \beta)=\tilde{G}^{>}(t)+$ $\mathcal{O}\left(\beta \mu_{\pi}\right)$ and neglect the nonperiodic terms, so that we end up with $\tilde{J}_{1} \simeq \int d^{3} \vec{x} \int_{0}^{\beta} d \tau \tilde{\Delta}_{T}^{4}(\tau, \vec{x})$ and $\tilde{J}_{2} \simeq$ $\int d^{3} \vec{x} \int_{0}^{\beta} d \tau\left(\partial_{\mu} \tilde{\Delta}_{T}(\tau, \vec{x}) \partial^{\mu} \tilde{\Delta}_{T}(\tau, \vec{x})\right)^{2}$. This approximation simplifies considerably the renormalization of this graph, since now we can follow the same steps as in [31]. First we separate $\tilde{\Delta}(\tau, \vec{x})=\tilde{G}^{>}(-i \tau, \vec{x})=\tilde{\Delta}(\tau, \vec{x})^{T=\mu_{\pi}=0}+$ $\delta \tilde{\Delta}(\tau, \vec{x})$ using the representation (B10) and (B11). The divergent contributions in the integrals (40) are then contained in the $\left(\delta \tilde{\Delta} \tilde{\Delta}^{0}\right)^{2}, \delta \tilde{\Delta}\left(\tilde{\Delta}^{0}\right)^{3}$, and $\left(\tilde{\Delta}^{0}\right)^{4}$ terms and can be renormalized with the same counterterms as in [31] replacing the $g_{0,1}$ by $\tilde{g}_{0,1}$. The finite part of the $\tilde{J}_{1,2}$ integrals can be evaluated numerically. A crucial point is that this approximation is consistent, as far as renormalization is concerned, with our previous evaluation of the $\tilde{z}_{8 \text { abde }}$ diagrams since the divergent parts of the terms proportional to $\tilde{g}_{0}^{2}, \tilde{g}_{0} \tilde{g}_{1}$, and $\tilde{g}_{1}^{2}$ arising from the $\tilde{J}_{1,2}$ integrals cancel exactly with those coming from the other four diagrams, while the terms proportional to $\tilde{g}_{1}$ add together to renormalize the physical pion mass according to the definition

$$
m_{\pi}=-\lim _{T \rightarrow 0^{+}} T \log \tilde{P}\left(T, \mu_{\pi}=0\right) .
$$

In addition, as it happens for $\mu_{\pi}=0$, this ensures that neither the tree-level constants from $\mathcal{L}_{6}$ nor the $T, \mu_{\pi}$ independent renormalization constants needed to render $\tilde{J}_{1,2}$ finite appear in the final expression for the free energy once it is expressed in terms of $m_{\pi}$. We remark that with our representation, not only is the renormalization procedure consistent, but the final answer for the full $\mathcal{O}\left(T^{8}\right)$ contribution amounts to replace $n\left(E_{p}\right) \rightarrow n\left(E_{p}-\mu_{\pi}\right)$ in all the spatial momentum integrals, without dealing with unnatural terms, like those discussed in Sec. III.

After the previous detailed evaluation, we arrive finally to a finite expression for the free energy, suitable for numerical evaluation, with the approximations discussed above implying that the $\mathcal{O}\left(T^{8}\right)$ corrections are reliable only for small $\mu_{\pi}$. From this expression we proceed to present our results for the $\mu_{\pi}$ dependence of several relevant observables.

\section{B. Results for thermodynamical observables}

From the energy density, we obtain the quark condensate (the order parameter of the chiral transition), the entropy density, and the pion number density in the standard way:

$$
\begin{gathered}
\langle\bar{q} q\rangle\left(T, \mu_{\pi}\right)=\langle\bar{q} q\rangle(0,0)\left[1+\frac{c}{f^{2}} \frac{\partial \tilde{P}\left(T, \mu_{\pi}\right)}{\partial m_{\pi}^{2}}\right], \\
\tilde{s}\left(T, \mu_{\pi}\right)=\frac{\partial \tilde{P}\left(T, \mu_{\pi}\right)}{\partial T}, \\
\tilde{n}\left(T, \mu_{\pi}\right)=\frac{\partial \tilde{P}\left(T, \mu_{\pi}\right)}{\partial \mu_{\pi}},
\end{gathered}
$$

where $c=1-m^{2}\left(4 \bar{h}_{1}+\bar{l}_{3}-1\right) /\left(32 \pi^{2} f^{2}\right)+\mathcal{O}\left(m^{4}\right)$.

We plot our results in Fig. 5. The first feature we observe is that the $\mathcal{O}\left(T^{6}\right)$ and the ideal gas curves are very close to one another for all the range of temperatures and chemical potentials shown. Sizable differences due to the interactions only show up numerically when including the $\mathcal{O}\left(T^{8}\right)$. 

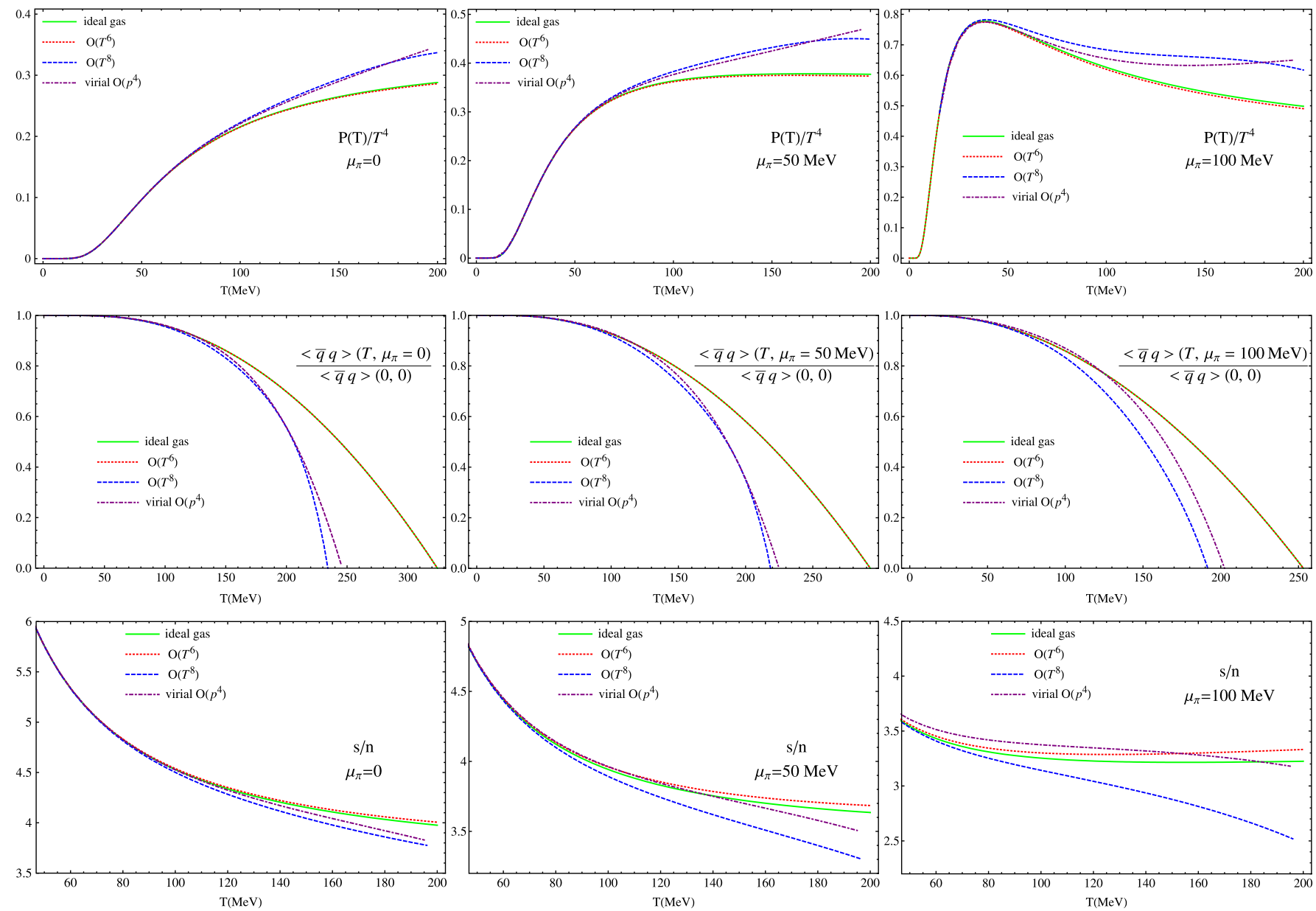

FIG. 5 (color online). Results for the pressure, quark condensate, and entropy over density ratio at different chemical potentials and to different orders in the ChPT interactions.

This is a also a feature of the $\mu_{\pi}=0$ calculation [31]. For instance, in the chiral limit $\left(m_{\pi}=0\right)$ and for $\mu_{\pi}=0$, the $\mathcal{O}\left(T^{6}\right)$ in (33) vanishes identically, while the $\mathcal{O}\left(T^{8}\right)$ survives, producing conformally anomalous contributions to the pressure [33]. In Fig. 5 we also compare our results with the virial gas approach [14], where the pressure can be written at low pion density in terms of the pion scattering phase shifts. In the curves shown in Fig. 5, the phase shifts have been calculated perturbatively to $\mathcal{O}\left(p^{4}\right)$ in ChPT and using the same set of low-energy constants as for our perturbative results with the approach of the present paper. We see that our $\mathcal{O}\left(T^{8}\right)$ results with $\mu_{\pi} \neq 0$ lie reasonably close to the virial result, at least for not very high $\mu_{\pi}$. This is a good consistency check of our present approach.

Another general feature that we observe in the curves is that the effect of the pion chemical potential is always to increase thermal effects. Effectively, it acts similar to a reduction of the effective pion mass [this is more accurate for $T \ll m_{\pi}$ where the typical momenta in the distribution functions are $\left.p=\mathcal{O}\left(\sqrt{T / m_{\pi}}\right)\right]$ and therefore for fixed $T$, the results for increasing $\mu_{\pi}$ go qualitatively in the same direction as for increasing $T$ with $\mu_{\pi}=0$. For instance, we see that the pressure increases for increasing $\mu_{\pi}$ and approaches faster with $T$ the asymptotic limit $P \sim$ $\pi^{2} T^{4} / 30$ [31] expected in the chiral limit $\left(T \gg m_{\pi}\right.$, $\mu_{\pi}$ ). The effect of interactions is also to increase the pressure, producing additive contributions in the ChPT expansion.

The curves for the quark condensate show that the chiral restoration temperature goes down for $\mu_{\pi} \neq 0$. This is also a consequence of the above discussed qualitative behavior, since the system for $\mu_{\pi} \neq 0$ is closer to chiral restoration. With the numerical values we get, we see that if chemical freeze-out takes place for temperatures below the chiral phase transition, then we do not expect to see any change in the value of $T_{c}$. On the contrary if $T_{\text {chem }}>T_{c}$ (which is less likely with the available experimental information), we would expect a reduction in $T_{c}$ compared with the estimates taking $\mu_{\pi}=0$.

It becomes clear from our discussion in Sec. II that incorporating additional physical requirements allowing one to describe $\mu_{\pi}(T)$ is crucial in our approach, in order to be consistent with the chemical nonequilibrium evolution. In this sense, a very interesting observable is the ratio 
of entropy density to pion density, also plotted in Fig. 5. It has been pointed out $[2,3,8]$ that on general grounds one expects this ratio to remain almost constant during the expansion. This is the isentropic expansion approximation, which is exact in the high $T$ limit $T \gg m_{\pi}, \mu_{\pi}$ for the ideal gas. We remark that we are restricting here to the gas of pions. If heavier degrees of freedom are included, such as the $\rho$, one has to account for the total number of pions $\bar{n}_{\pi}=n_{\pi}+2 n_{\rho}+\cdots$ which includes those "stored" in the $\rho$ if the channel $\rho \rightarrow \pi \pi$ is considered as the only source of pion number changing, and similarly with other resonances (see details in $[2,8]$ ). The idea is then that by fixing $s / n$ to a given value at the chemical freeze-out temperature $T_{\text {chem }}$, where $\mu_{\pi}=0$, going down in the temperature scale one can keep $s / n$ fixed by increasing $\mu_{\pi}$, as can be seen in Fig. 5. This provides the isentropic dependence $\mu_{\pi}(T)$, which is given in [2,8] for the ideal gas approximation. We plot in Fig. 6 the isentropic curves $\mu_{\pi}(T)$ with a reference value $s / n=4$, for which $T_{\text {chem }} \simeq$ $190 \mathrm{MeV}$ for the ideal gas. The obtained curves follow a roughly linear behavior, as expected phenomenologically [3]. The most significant effect we observe is the reduction of $T_{\text {chem }}$ when $\mathcal{O}\left(T^{8}\right)$ or virial interactions are included. This is a very natural effect since, as we have discussed in previous sections, that order in the interaction is the one where particle-changing processes begin to be relevant and drive the system back to chemical equilibration. The virial curve lies reasonably close to our perturbative $\mathcal{O}\left(T^{8}\right)$ since the two approaches differ significantly only for rather high values of $\mu_{\pi}$ and $T$, which are not reached along the curve $\mu_{\pi}(T)$. In fact, in the isentropic evolution our $\mathcal{O}\left(T^{8}\right)$ approach is better justified since $\mu_{\pi}(T) \ll T, m_{\pi}$. We also remark that the same effect of faster equilibration is seen when comparing the curves of the ideal pion gas with that of the ideal pion plus resonances gas, as done in [2]. One can check that the curves in that paper for $s / n$ as a function of $T$ for different $\mu_{\pi}$ are systematically lower when including resonances, as in our case in Fig. 5 when

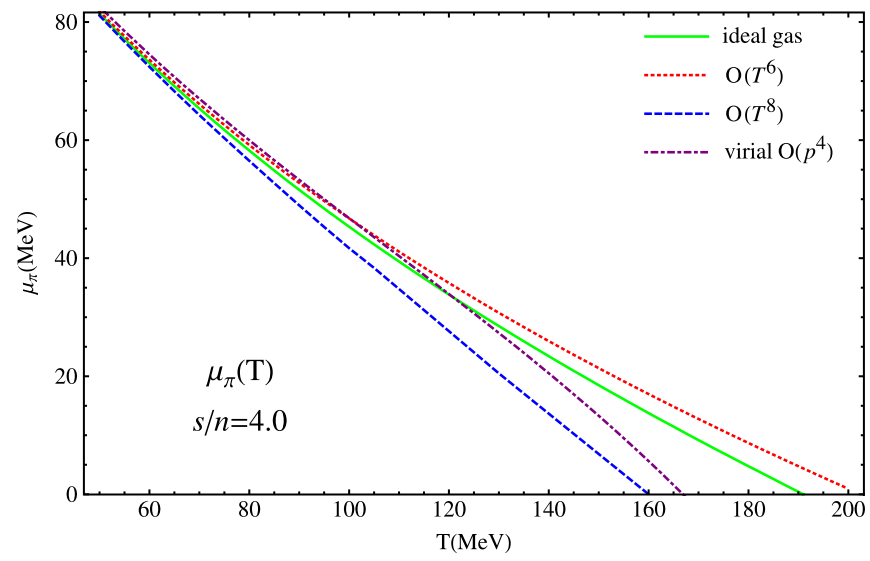

FIG. 6 (color online). Dependence of $\mu_{\pi}(T)$ in the isentropic approximation, with the fixed value $s / n=4$. including the $\mathcal{O}\left(T^{8}\right)$ or in the virial case and therefore the free pion and resonance gas equilibrates faster, which is the feature that we are able to reproduce here including higherorder pion interactions.

\section{Self-energy: Pion thermal mass and width}

Within the real-time formalism developed in Sec. III B, we can calculate the pion self-energy for $\mu_{\pi} \neq 0$, whose leading-order corrections to its real and imaginary parts are given by the diagrams in Figs. 3(a) and 3(b), respectively, with all vertices coming from the $\mathcal{L}_{2}$ Lagrangian.

It is important to remark that when nonequilibrium distributions are considered, as is our case here, it has been pointed out that additional $\delta^{2}$-like or pinching-pole ill-defined contributions arise [25], which should be regularized keeping a nonzero particle width. We will discuss the role of those contributions in the last part of this section.

Consider first Fig. 3(a). It includes a contribution with a constant vertex proportional to $m^{2} \tilde{G}(0) \partial \tilde{D}_{11}(p) / \partial m^{2}$ which directly renormalizes the pion mass, following the prescriptions explained in Sec. III B, and derivative vertices, which contribute either proportional to $\square \tilde{G}(0)=-m^{2} \tilde{G}(0) \quad$ (mass renormalization) or as $\tilde{G}(0) p^{2} \partial \tilde{D}_{11}(p) / \partial m^{2}=\tilde{G}(0)\left(\tilde{D}_{11}(p)+m^{2} \partial \tilde{D}_{11}(p) / \partial m^{2}\right)$ (mass and wave function renormalization). One can then follow similar steps as in the standard derivation of the thermal corrections to the pion self-energy to this order $[10,34]$, the wave function renormalization being directly related to the thermal $f_{\pi}$ through the usual definition in terms of the residue of the axial-axial current correlator. The ultraviolet divergences arising in the calculation are absorbed by the renormalization of the low-energy constants $l_{3}$ and $l_{4}$. We finally obtain

$$
\begin{gathered}
m_{\pi}^{2}\left(T, \mu_{\pi}\right)=m_{\pi}^{2}+\frac{m^{2}}{2 f^{2}} \tilde{g}_{1}\left(m, T, \mu_{\pi}\right)+\mathcal{O}\left(m^{4}\right), \\
f_{\pi}^{2}\left(T, \mu_{\pi}\right)=f_{\pi}^{2}-2 \tilde{g}_{1}\left(m, T, \mu_{\pi}\right)+\mathcal{O}\left(m^{4}\right),
\end{gathered}
$$

with $m_{\pi}$ and $f_{\pi}$ the $T=\mu_{\pi}=0$ physical values given in (34) and (35) in terms of $m$ and $f$ to this order.

Taking into account now the corrections to the quark condensate to the same chiral order, i.e., $\mathcal{O}\left(T^{6}\right)$, which is given from (42) and (33) using (B35):

$$
\begin{aligned}
\langle\bar{q} q\rangle\left(T, \mu_{\pi}\right)= & \langle\bar{q} q\rangle(0,0)\left[1-\frac{3}{2 f^{2}} \tilde{g}_{1}\left(m, T, \mu_{\pi}\right)\right] \\
& +\mathcal{O}\left(T^{8}\right),
\end{aligned}
$$

we obtain that the Gell-Mann-Oakes-Renner (GOR) relation [35] holds also for $\mu_{\pi} \neq 0$ to this order (one-loop ChPT):

$$
\frac{f_{\pi}^{2}\left(T, \mu_{\pi}\right) m_{\pi}^{2}\left(T, \mu_{\pi}\right)}{\langle\bar{q} q\rangle\left(T, \mu_{\pi}\right)}=\frac{f_{\pi}^{2}(0,0) m_{\pi}^{2}(0,0)}{\langle\bar{q} q\rangle(0,0)}=-m_{q} .
$$


The GOR relation in terms of thermal quantities at $T \neq$ $0, \mu_{\pi}=0$ had been verified to one loop in [36]. To this order, the thermal mass varies little, also at $\mu_{\pi} \neq 0$ (see below) so that the evolution of $f_{\pi}$ follows that of the quark condensate and both behave as order parameters. However, beyond one loop, the GOR does not hold for thermal quantities [36].

Another important observation is that the shift (45) in the mass to this order can be written, as in the $\mu_{\pi}=0$ case [10] in terms of the elastic pion-pion forward scattering amplitude, from (B31):

$$
\begin{aligned}
m_{\pi}^{2}\left(T, \mu_{\pi}\right)-m_{\pi}^{2}= & -\int \frac{d^{3} \vec{p}}{(2 \pi)^{3}} \frac{1}{2 E_{p}} \frac{1}{e^{\beta\left(E_{p}-\mu_{\pi}\right)}-1} \\
& \times \operatorname{Re}\left[T_{\pi \pi}^{f}\left(s=\left(E_{p}+m_{\pi}\right)^{2}-|\vec{p}|^{2}\right)\right],
\end{aligned}
$$

where $E_{p}^{2}=m_{\pi}^{2}+|\vec{p}|^{2}$ and $T_{\pi \pi}^{f}(s)$ is the isospin averaged forward scattering amplitude:

$$
\begin{aligned}
T_{\pi \pi}^{f}(s) & \equiv T_{\pi \pi}(s, 0, u)=\frac{1}{3} \sum_{I=0}^{2}(2 I+1) T_{I}(s, 0, u) \\
& =\frac{32 \pi}{3} \sum_{I=0}^{2} \sum_{J=0}^{\infty}(2 I+1)(2 J+1) t_{I J}(s) \\
& \simeq \frac{32 \pi}{3}\left[t_{00}(s)+9 t_{11}(s)+5 t_{20}(s)\right] \\
& =-\frac{m^{2}}{f^{2}}+\mathcal{O}\left(s^{2}, m^{4}\right),
\end{aligned}
$$

where the last expression is the lowest order $\mathcal{O}\left(p^{2}\right)$ (treelevel diagrams with $\mathcal{L}_{2}$ vertices); $T_{I}(s, t, u)$ are the projections of the scattering amplitude with definite isospin $I$; $s$, $t$, and $u$ are the Mandelstam variables satisfying $s+t+$ $u=4 m_{\pi}^{2}$; and $t_{I J}$ are the partial waves, defined in the center of mass frame with definite isospin $I$ and angular momentum $J$. We follow the conventions in [30]. In the previous expression, we have included only the partial waves with lowest angular momentum $J \leq 1$. Those with $J>1$ are negligible for $\sqrt{s}$ below inelastic thresholds, such as the $K \bar{K}$ one, and for the temperatures involved here [10].

The result in (49) is the generalization to $\mu_{\pi} \neq 0$ of the formula relating the shift in the self-energy with the density of states and the scattering amplitude to lowest order in the density (dilute gas regime) [10]. These types of relations were first derived by Luscher [37] studying finite-volume corrections. A very interesting point is that it admits a natural extension [10] by considering (in the dilute gas regime) not only the perturbative tree-level $\mathcal{O}\left(p^{2}\right)$ amplitude, but also higher orders, including unitarized amplitudes. In the latter case, unitarized partial waves $t_{I J}^{U}(s)$ for $\pi \pi$ scattering can be constructed to exactly satisfy the unitarity relation:

$$
\operatorname{Im} t_{I J}^{U}(s)=\sqrt{1-\frac{4 m_{\pi}^{2}}{s}}\left|t_{I J}^{U}\right|^{2}
$$

matching at the same time the perturbative ChPT expansion and providing expressions that can be analytically continued to the complex $s$ plane. All these features are satisfied by the inverse amplitude method (IAM) scattering amplitudes [38] which reproduce scattering data up to $\sqrt{s} \sim 1 \mathrm{GeV}$ and all the low-lying resonances, which in the pure pion case considered here reduce to the $\rho(770)$ and the $f_{0}(600)$ or $\sigma$. Recall that the ChPT amplitudes satisfy the unitarity relation (51) only perturbatively order by order, violating the unitarity bounds for higher energies and thus not being able to reproduce resonances.

In Fig. 7 we show our results for the thermal mass, considering $\mathcal{O}\left(p^{2}\right), \mathcal{O}\left(p^{4}\right)$ and IAM unitarized amplitudes in (49). We have used the same set of low-energy constants as in our previous calculations, i.e., the $\bar{l}_{i}$ given at the beginning of Sec. IVA. For the case of the unitarized amplitudes, this set is adequate to compare with the perturbative ChPT expressions, although it does not give the best results for the mass and width of the resonances generated with the IAM. We have checked that our results do not change qualitatively by changing for instance to the set given in [39], which gives better physical values for the $\rho, f_{0}(600)$ mass and width.

Our results show that the leading order, the $\operatorname{ChPT} \mathcal{O}\left(p^{2}\right)$ given by the tadpole diagram in Fig. 3(a), produces a thermal mass slightly increasing with temperature and chemical potential. However, including the $\mathcal{O}\left(p^{4}\right)$ or unitarized corrections to the amplitude, the mass tends to decrease significantly with $T$ and $\mu_{\pi}$. Our results at $\mu_{\pi}=$ 0 agree with [10]. The difference between the $\mathcal{O}\left(p^{4}\right)$ and the unitarized curve is not very relevant here. Our $\mathcal{O}\left(p^{2}\right)$ curve agrees reasonably with a linear-sigma model calculation [40] which agrees with ChPT to this order at $\mu_{\pi}=0$ and where the chemical potential is introduced in analogy with the charged scalar field case.

These results suggest an interesting scenario: the pion system could undergo Bose-Einstein (BE) condensation driven by the dropping of the thermal mass by interactions. Recall that we are dealing with BE condensation of both neutral and charged pions, since we are considering an electrically neutral system with finite pion density. The physical situation is then different from the charged pion or kaon condensation taking place in nuclear matter [41] or isospin chemical potential [20] scenarios, although the dropping of the effective mass takes place also in the former. $\mathrm{BE}$ condensation for pion number and its possible phenomenological consequences in heavy-ion collisions has been extensively studied in the literature [42]. Among the observable consequences are the anomalous enhancement of the low- $p_{T}$ pion spectrum and of number fluctuations in high multiplicity events. 

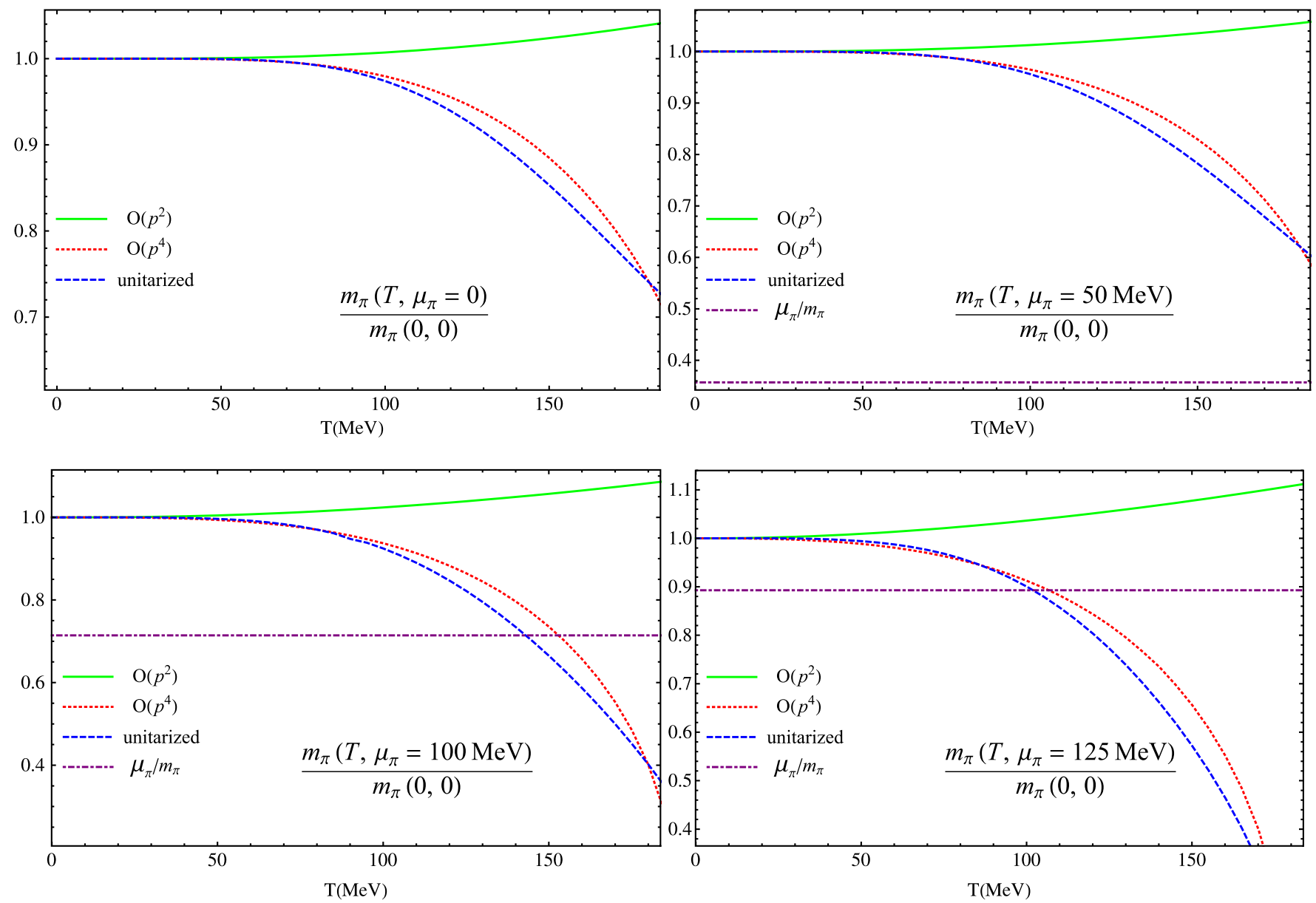

FIG. 7 (color online). Results for the thermal mass dependence on temperature and pion chemical potential, considering different orders in the scattering amplitude in (49).

In our grand-canonical interacting framework we can describe the corrections to $\mathrm{BE}$ condensation due to pion interactions. In the standard free case, the BE limit is reached when $\mu_{\pi} \rightarrow m_{\pi}$ from below (by definition the system is below the condensed phase). Those values for the pion chemical potential seem too high compared with those measured in heavy-ion collisions at thermal freezeout $T_{\text {ther }} \sim 100 \mathrm{MeV}[2,3,5]$. In other words, the required
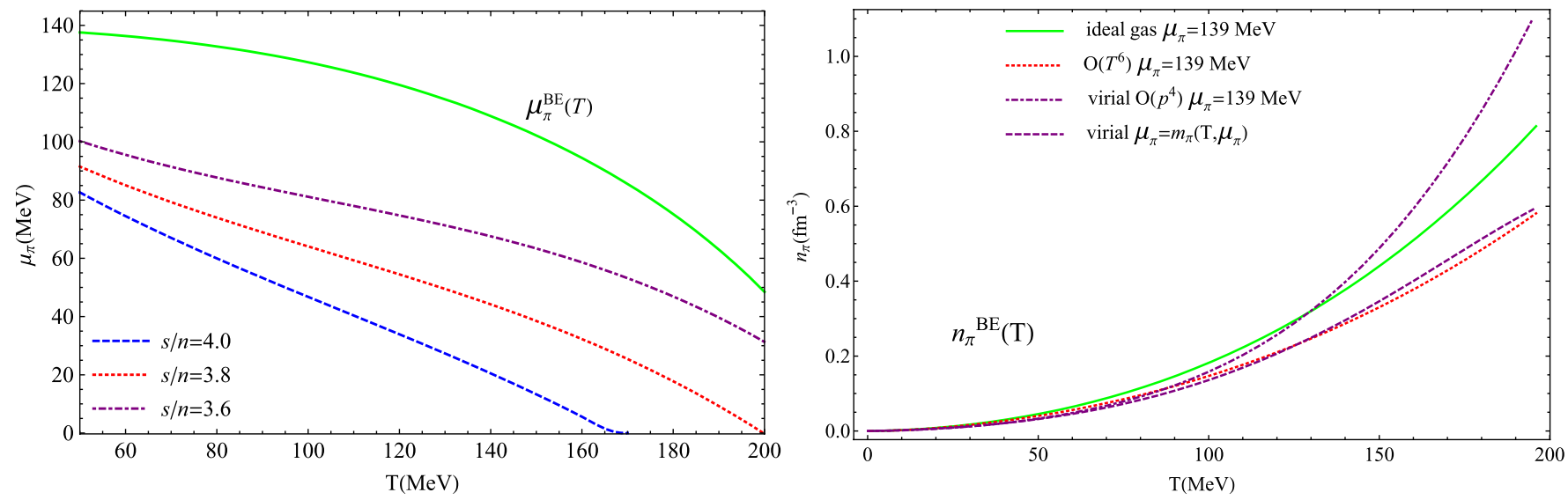

FIG. 8 (color online). Bose-Einstein condensation lines. Left panel: the curve $\mu_{\pi}^{\mathrm{BE}}=m_{\pi}\left(T, \mu_{\pi}\right)$ with the thermal mass from the $\mathcal{O}\left(p^{4}\right)$ amplitudes, compared to the isentropic expansion curves for the virial case to the same order and for different $s / n$ values. Right panel: pion density versus temperature in the BE limit $\mu_{\pi} \rightarrow m_{\pi}^{-}$for different orders in the interaction, compared to the ideal gas and to the virial case with thermal mass. 
pion densities for $\mathrm{BE}$ condensation might not be reached. However, if the effective particle mass $m_{\pi}\left(T, \mu_{\pi}\right)$ drops by interactions among the medium constituents, the value $\mu_{\pi}=m_{\pi}\left(T, \mu_{\pi}\right)$ would be reduced. We show that line in Fig. 7. In Fig. 8 (left panel) the resulting $\mu_{\pi}^{\mathrm{BE}}(T)=$ $m_{\pi}\left(T, \mu_{\pi}^{\mathrm{BE}}(T)\right)$ curve is represented and compared with the isentropic curves corresponding to different values of $s / n$. We see that the BE curve thus defined lies not very far from the isentropic approach and the expected phenomenological values. Those curves correspond to the $\mathcal{O}\left(p^{4}\right)$ amplitudes, both for the thermal mass and for $s$ and $n$ (in the virial approach). In Fig. 8 (right panel) we show also the density-temperature curves corresponding to $\mu_{\pi} \rightarrow m_{\pi}^{-}$ for different orders in the interaction. The $\mathcal{O}\left(T^{6}\right)$ allows for lower density values, but the virial contribution points in the opposite direction. We also show the curve corresponding to the BE limit by lowering of the mass, as we have just explained, for the same virial approach, which produces a considerable lowering of the required densities. In any case, the corrections due to interactions are small near thermal freeze-out. We also remark that some of our previous results, including those regarding $\mathrm{BE}$ condensation by mass reduction, rely on the validity of the dilute gas regime, for instance when using (49), but corrections might be important for temperatures close to the chiral transition or chemical potentials close to $m_{\pi}$.

Finally, we turn to the calculation of the leading-order imaginary part of the pion self-energy, given in ChPT by Fig. 3(b). This is the leading-order contribution to the thermal collisional width $\Gamma_{p}=-\operatorname{Im} \Sigma_{R}\left(E_{p},|\vec{p}|\right) /\left(2 E_{p}\right) \ll$ $E_{p}$ with $E_{p}=\sqrt{|\vec{p}|^{2}+m_{\pi}^{2}}$ and $\Sigma_{R}$ the retarded selfenergy, which defines the dispersion relation $p^{2}=m_{\pi}^{2}+$ $\Sigma_{R}\left(p_{0},|\vec{p}| ; T\right)$.

As we have commented on in Sec. III B, we will evaluate the retarded correlator in the real-time formalism, following the circling rules in [28], which also apply to the $\mu_{\pi} \neq$ 0 case. Applying those rules to the diagram in Fig. 3(b) we have

$$
\begin{aligned}
\operatorname{Im} \Sigma_{R}\left(p_{0},|\vec{p}|\right) & =-\frac{1}{2}\left[\tilde{H}^{>}\left(p_{0},|\vec{p}|\right)-\tilde{H}^{<}\left(p_{0},|\vec{p}|\right)\right] \\
& =-\frac{i}{2}\left[\Sigma_{21}\left(p_{0},|\vec{p}|\right)-\Sigma_{12}\left(p_{0},|\vec{p}|\right)\right],
\end{aligned}
$$

where $\tilde{H}^{>(<)}$are obtained by using for the three internal lines in the diagram the $\tilde{G}^{>(<)}$RTF propagators. With the usual RT self-energy definition [24] and our convention for the $D_{12}, D_{21}$ propagators given in Sec. III B, we have $H^{>}=i \Sigma_{21}$ and $H^{<}=i \Sigma_{12}$ for the diagram in Fig. 3(b), since, once a particular choice of $i j$ indices $(i, j=1,2)$ has been made for the two vertices in that diagram, the three internal lines carry the same $i j$ combination.

Now, according to our discussion in Sec. III B and in Appendix B:

$$
\begin{aligned}
\tilde{G}^{>}(k) & =2 \pi \delta\left(p_{0}^{2}-E_{p}^{2}\right)\left[\theta\left(p_{0}\right)+n\left(\left|p_{0}\right|-\mu_{\pi}\right)\right] \\
& =e^{\tilde{\beta}_{k} k_{0}} \tilde{G}^{<}(k)=e^{\beta\left[k_{0}-\mu_{\pi} \operatorname{sgn}\left(k_{0}\right)\right]} \tilde{G}^{<}(k),
\end{aligned}
$$

so that we get for the thermal width:

$$
\begin{aligned}
\Gamma_{p}\left(T, \mu_{\pi}\right)= & \frac{1}{4 E_{p}} \int \prod_{i=1}^{3} \frac{d^{4} k_{i}}{(2 \pi)^{4}} \Lambda\left(k_{1}, k_{2}, k_{3}, p\right) \\
& \times \tilde{G}^{>}\left(-k_{1}\right) \tilde{G}^{>}\left(k_{2}\right) \\
& \times \tilde{G}^{>}\left(k_{3}\right)\left(1-e^{-\beta\left[E_{p}-\mu_{\pi} f\left(k_{1}, k_{2}, k_{3}\right)\right]}\right)(2 \pi)^{4} \\
& \times \delta\left(E_{p}+k_{1}^{0}-k_{2}^{0}-k_{3}^{0}\right) \\
& \times \delta^{(3)}\left(\vec{p}+\vec{k}_{1}-\vec{k}_{2}-\vec{k}_{3}\right),
\end{aligned}
$$

where $k_{1,2,3}$ label the three internal lines, $\Lambda$ is the squared vertex function coming from the $\mathcal{L}_{2}$ Lagrangian, and

$$
f\left(k_{1}, k_{2}, k_{3}\right)=\operatorname{sgn}\left(k_{2}^{0}\right)+\operatorname{sgn}\left(k_{3}^{0}\right)-\operatorname{sgn}\left(k_{1}^{0}\right) .
$$

Recall that in the $\mu_{\pi}=0$ case, the $f$ term is absent so that one ends up with a prefactor $e^{-\beta E_{p}}-1=$ $1 /\left(1+n\left(E_{p}\right)\right)$ in the thermal width. The natural expectation from replacing just the distribution function $n \rightarrow \tilde{n}_{p}$ for $\mu_{\pi} \neq 0$ would be then $E_{p} \rightarrow E_{p}-\mu_{\pi}$ in that factor, as well as the modifications of the internal distribution functions $n\left(E_{i}\right) \rightarrow n\left(E_{i}-\mu_{\pi}\right)$, where $E_{i}$ is short for $E_{k_{i}}$. This is indeed the result found in [9] derived from kinetic theory. In our case, it is not obvious that the answer is the same, since the function $f$ above is not equal to 1 for the eight possible combinations of signs of the three internal $k_{i}^{0}$. We denote them by $s_{1} s_{2} s_{3}$, with $s_{i}=\operatorname{sgn}\left(k_{i}^{0}\right)$. Now, we take into account that the $\delta$ functions in each of the internal lines put them on shell, i.e., $k_{i}^{0}= \pm E_{i}$ and global energymomentum conservation in the diagram imposed by the $\delta$ functions in (54). Thus, the combination +-- giving $f=-3$ is excluded by energy conservation $E_{p}+E_{1}>$ $0>-E_{2}-E_{3}$. On the other hand, from three-momentum conservation and the on-shell conditions we have that for any combination it should hold $A=B$, where we denote $A \equiv E_{p}^{2}+E_{1}^{2}-E_{2}^{2}-E_{3}^{2}$ and $B \equiv 2\left(\vec{k}_{2} \cdot \vec{k}_{3}-\vec{p} \cdot \vec{k}_{1}\right)$ and, in addition $-C \leq B \leq C$ with $C=2\left(p k_{1}+k_{2} k_{3}\right)$, and where $k_{i}, p$ are short for $\left|\vec{k}_{i}\right|$ and $|\vec{p}|$, respectively. Therefore, the case $++-(f=-1)$ is also excluded, since for that combination $E_{p}+E_{1}=E_{2}-E_{3}$ so that $A=-2\left(E_{p} E_{1}+E_{2} E_{3}\right)<-C$. The same reason excludes $+-+(f=-1)$. Combinations $-++(f=3)$ and $---(f=-1)$ give $A=2\left(E_{p} E_{1}+E_{2} E_{3}\right)>C$ and are thus excluded as well. Therefore, the only combinations remaining are,+++-+- , and --+ , the three of them giving $f=1$ and the same contribution from the vertices as for $\mu_{\pi}=0$, given in [10].

It is not difficult to repeat the above analysis, now with the external energy $p_{0}=-E_{p}$. In that case, every combination of relative signs between the $E_{i}$ is obtained from the previous case by flipping the three $s_{i}$, the $A, B$, and $C$ 
functions being independent of the sign of $p_{0}$. Thus, applying the same arguments, the only surviving combinations are now,---+-+ , and ++- , the three of them giving $f=-1$. Therefore, what we have proven in terms of the 12 and 21 components of the self-energy for this diagram is

$$
\begin{aligned}
\Sigma_{21}^{6 b}\left(p_{0}= \pm E_{p}, p\right) & =e^{\beta\left(p_{0}-\mu_{\pi} \operatorname{sgn}\left(p_{0}\right)\right)} \Sigma_{12}^{6 b}\left(p_{0}= \pm E_{p}, p\right) \\
& =e^{\tilde{\beta}_{p} p_{0} \Sigma_{12}^{6 b}\left(p_{0}= \pm E_{p}, p\right)}
\end{aligned}
$$

which is the usual equilibrium relation with $\beta \rightarrow \beta_{p}$. This relation will be of use in the discussion at the end of this section about possible higher-order corrections related to pinching poles.

In conclusion, the result (54) we find with our diagrammatic method is the same as in kinetic theory [9], which, after relabeling $k_{1} \leftrightarrow-k_{3}$ in -+- and $k_{1} \leftrightarrow-k_{2}$ in --+ and performing the three integrals in $k_{i}^{0}$ using the on-shell $\delta$ functions, can be written as

$$
\begin{aligned}
\Gamma_{p}\left(T, \mu_{\pi}\right)= & \frac{1}{8 E_{p}} \frac{1}{1+n\left(E_{p}-\mu_{\pi}\right)} \int \prod_{i=1}^{3} \frac{d^{3} k_{i}}{(2 \pi)^{3} 2 E_{i}} \\
& \times n\left(E_{1}-\mu_{\pi}\right)\left[1+n\left(E_{2}-\mu_{\pi}\right)\right] \\
& \times\left[1+n\left(E_{3}-\mu_{\pi}\right)\right]\left|T_{\pi \pi}(s, t)\right|^{2}(2 \pi)^{4} \\
& \times \delta\left(E_{p}+E_{1}-E_{2}-E_{3}\right) \\
& \times \delta^{(3)}\left(\vec{p}+\vec{k}_{1}-\vec{k}_{2}-\vec{k}_{3}\right),
\end{aligned}
$$

where $T_{\pi \pi}$ is the isospin averaged elastic pion scattering amplitude with $s=\left(E_{p}+E_{1}\right)^{2}-\left|\vec{p}+\vec{k}_{1}\right|^{2}$, and $t=$ $\left(E_{p}-E_{2}\right)^{2}-\left|\vec{p}-\vec{k}_{2}\right|^{2}$.

Taking now the dilute gas regime in the previous expression, which amounts to neglect all the Bose-Einstein functions $n \ll 1$ except $n\left(E_{1}-\mu_{\pi}\right)$, gives rise to the extension of Luscher's formula in terms of the forward scattering amplitude, as in (49) but now for the imaginary part of the self-energy through the pion thermal width (which vanishes at $T=0$ ):

$$
\begin{aligned}
\Gamma_{p}^{D G}\left(T, \mu_{\pi}\right)= & \frac{1}{2 E_{p}} \int \frac{d^{3} \vec{k}}{(2 \pi)^{3}} n\left(E_{k}-\mu_{\pi}\right) \\
& \times \frac{\sqrt{s\left(s-4 m^{2}\right)}}{2 E_{k}} \sigma_{\pi \pi}(s) \\
= & \frac{1}{2 E_{p}} \int \frac{d^{3} \vec{k}}{(2 \pi)^{3} 2 E_{k}} n\left(E_{k}-\mu_{\pi}\right) \operatorname{Im} T_{\pi \pi}^{f}(s),
\end{aligned}
$$

where we have relabeled $k_{1} \rightarrow k$ and $\sigma_{\pi \pi}$ is the total $\pi \pi$ cross section

$$
\begin{aligned}
\sigma_{\pi \pi}(s) & =\frac{32 \pi}{3 s} \sum_{I J}(2 I+1)(2 J+1)\left|t_{I J}(s)\right|^{2} \\
& =\frac{1}{\sqrt{s\left(s-4 m^{2}\right)}} \operatorname{Im} T_{\pi \pi}^{f}(s),
\end{aligned}
$$

where the last line is the optical theorem, following from exact unitarity (51).

We remark that our final results both for the real and imaginary parts of the self-energy to this order correspond to the replacement $n \rightarrow \tilde{n}$ evaluated at positive energies. This is not only natural from the kinetic theory viewpoint but it is also formally obtained by performing such replacement in the analytically continued $\mu_{\pi}=0$ ITF selfenergies.

The thermal width is of phenomenological relevance, since it enters directly in the calculation of transport coefficients in the meson gas $[33,43]$. It is then important to estimate pion chemical potential effects in the width during the phase of chemical nonequilibrium, where the particle number is approximately conserved and transport phenomena can be described relying on the dominance of elastic collisions, which is also consistent with the dilute gas regime. On the other hand, in this regime the mean collision time defined for ultrarelativistic particles as $\tau=$ $1 /(2 \bar{\Gamma})[3,8,9]$ with the averaged width:

$$
\bar{\Gamma}\left(T, \mu_{\pi}\right)=\frac{\int d^{3} \vec{p} \Gamma_{p}\left(T, \mu_{\pi}\right) n\left(E_{p}-\mu_{\pi}\right)}{\int d^{3} \vec{p} n\left(E_{p}-\mu_{\pi}\right)},
$$

provides direct information about thermal relaxation. We represent $\tau$ in the dilute approach in Fig. 9, using the scattering amplitude in (58) to different orders, including the unitarized case. We use the same set of $\bar{l}_{i}$ constants as in the rest of the paper.

We see in the figure that the effects of correctly reproducing the energy behavior of the scattering amplitude is important for evaluating the collision time. In particular, the unitarized curve shows important differences with the perturbative ones in the temperature range shown. This was also noticed in [10] at $\mu_{\pi}=0$ and the importance of including unitarized corrections to the width for transport coefficients in the meson gas has been highlighted in $[33,43]$, for instance, regarding violations of AdS/CFT bounds for the shear viscosity over entropy ratio or correlations between the bulk viscosity and the conformal anomaly.

Another clear effect that we observe is the reduction of the mean time with the pion chemical potential, also observed in [9] with $\mathcal{O}\left(p^{2}\right)$ amplitudes. Physically, in the temperature regime where $\tau$ is much smaller than the typical plasma lifetime $(\sim 10 \mathrm{fm} / c)$, which at the same time is small compared to the inelastic collision time driving the system to chemical equilibrium, the system remains in thermal but not chemical equilibrium. From the estimates of the inelastic collision rates given for instance in [8] and the results in Fig. 9, this would happen 

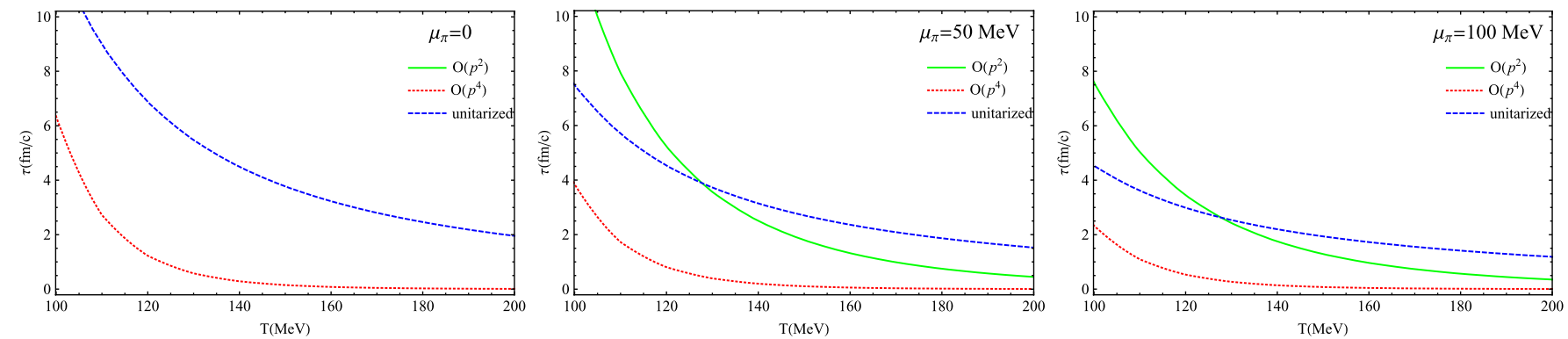

FIG. 9 (color online). Mean collision time in the elastic and dilute limits, considering different orders for the pion scattering amplitude and different values for the pion chemical potential.

at $\mu_{\pi}=0$ typically in the range $120 \mathrm{MeV}<T<$ $180 \mathrm{MeV}$. However, precisely in that regime, and as we have explained in this paper, $\mu_{\pi} \neq 0$, its typical values being given by the isentropic curve in Fig. 6, which means that the range of thermal equilibrium enlarges from below, from the commented reduction of $\tau$ with $\mu_{\pi}$. In fact, estimating the thermal freeze-out temperature $T_{\text {ther }}$ as that where this approximation ceases to be valid, i.e., where $\tau \sim 10 \mathrm{fm} / c$ (this type of dynamical condition has also been used in [1] to determine the freeze-out conditions) gives a shift in the thermal freeze-out temperature $\Delta T_{\text {ther }} \simeq-20 \mathrm{MeV}$ with respect to the $\mu_{\pi}=0$ case, following approximately the isentropic values in Fig. 6. In particular, using the unitarized results in Fig. 9 we obtain in this way $T_{\text {ther }} \simeq 95 \mathrm{MeV}$, close to experimental values.

Finally, let us comment on the pathological nonequilibrium terms found in [25] in a $g^{2} \phi^{4}$ context. Those terms are of the type of $\delta^{2}$ functions at the same point, or pinching singularities and therefore have to be regularized by keeping a nonzero particle width in the propagators, i.e., $\Gamma_{p} \neq 0$ in our case. The first nonvanishing term of this kind in the $g^{2} \phi^{4}$ theory is the three-loop diagram given in Fig. 2b of [25]. Note that formally this is an $\mathcal{O}\left(g^{6}\right)$ correction, while the diagrams we have considered here in Fig. 3, for which there are no such pathologies, would be $\mathcal{O}\left(g^{2}\right)$ and $\mathcal{O}\left(g^{4}\right)$, respectively, in that counting. Nevertheless, the argument in [25] is that those contributions are proportional to the inverse width $1 / \Gamma$ with $\Gamma=\mathcal{O}\left(g^{4}\right)$ and therefore could become of the same order as the leading ones. The form of such a leading pinching-pole term [25] in our case is

$$
\int d P \Delta_{R}(p) \Delta_{A}(p)\left\{\left[\left(1+\tilde{n}_{p}\left(p_{0}\right)\right] \Sigma_{12}^{6 b}-\Sigma_{21}^{6 b} \tilde{n}_{p}\left(p_{0}\right)\right\},\right.
$$

where the self-energy components of the diagram in Fig. 3(b) that we have analyzed above enter directly to this order and $\Delta_{R, A}$ denote the retarded/advanced propagators. Let us isolate the leading $\Gamma_{p} \rightarrow 0^{+}$behavior of the previous expression. The product $\Delta_{R} \Delta_{A}$ is the characteristic pinching-pole contribution appearing typically in diagrammatic calculations of transport coefficients $[33,43]$ and in the $\Gamma_{p} \rightarrow 0^{+}$behaves as

$$
\Delta_{R}(p) \Delta_{A}(p) \stackrel{\Gamma_{p} \rightarrow 0^{+}}{\approx} \frac{\pi}{2 E_{p} \Gamma_{p}} \delta\left(p_{0}^{2}-E_{p}^{2}\right)
$$

which puts on shell $\left(p_{0}= \pm E_{p}\right)$ the integrand of (61). Now, we recall the relation (56) we have derived for the diagram in Fig. 3(b), together with the properties of the "modified" distribution function, in particular, $1+$ $\tilde{n}_{p}\left(p_{0}\right)=e^{\tilde{\beta}_{p}} \tilde{n}_{p}\left(p_{0}\right)$. Altogether, this means that the leading-order pathological contribution (61) vanishes in our case in the $\Gamma_{p} \rightarrow 0^{+}$limit. There may be higher-order corrections of this kind, but in accordance with the power counting in [25], those would be subleading with respect to the contributions in Fig. 3 that we have analyzed in this section.

The previous analysis showing the absence of pathological terms is only valid to lowest order in those terms. Further conclusions can only be reached with a complete ChPT analysis of higher-order pinching-pole contributions, extending that in [25], which is beyond the scope of this work. Nevertheless, it is worth pointing out that the low- $T$ ChPT counting does not involve any coupling constant, which implies important differences with respect to the $g^{2} \phi^{4}$ one in the perturbative behavior of pinching diagrams at low $T$ [43]. In addition, the analysis of [25] shows that the nonequilibrium pathological terms are always proportional to $\delta n$, the deviation from the Bose-Einstein distribution function. Thus, in our case, we can use an argument similar to the one we invoked in Sec. IVA when dealing with the $\mathcal{O}\left(T^{8}\right)$ terms in the partition function. Namely, that in the ChPT counting, those diagrams are expected to be important for temperatures for which $\beta \mu(T) \sim \delta n \ll 1$, giving a further suppression. We finally remark that in the $\mu_{\pi} \neq 0$ scenario, the presence of nonvanishing pinching-pole terms have also their origin in the presence of particle-changing processes (see also our discussion in Sec. IVA). In fact, self-energy combinations of the form (61) enter directly in the Boltzmann equation describing the rate of particle number change [25]. The fact that the leading correction of that type (61) vanishes in our case seems to be related to the fact that the leading selfenergy corrections arising from the diagrams in Fig. 3 can always be expressed in terms only of the elastic $\pi \pi$ 
scattering amplitude, as we have discussed extensively throughout this section.

\section{CONCLUSIONS}

In this work we have developed a path-integral diagrammatic formalism in order to deal with chemical nonequilibrium effects in interacting scalar field theories, in the regime where particle number is approximately conserved. Within the theoretical framework of holomorphic path integrals and thermal field theory, we have derived the relevant Feynman rules for nonzero particle number chemical potential $\mu$, whose validity is restricted to the temperature regimes where one can neglect particlechanging processes. This derivation in the interacting case is original to this paper, to the best of our knowledge.

We have addressed some subtleties related to the choice of contour in complex times, leading to the extension of real- and imaginary-time formalisms at $\mu \neq 0$. We have shown that the consistent formulation is the real-time one, in agreement with other nonequilibrium formulations. The imaginary-time formalism can lead to spurious contributions, related to the loss of periodicity or global KMS conditions. These problems are not present in the realtime formalism, once a proper energy representation for the propagators is chosen, in accordance with the standard $\mu=0$ choice. In addition, following previous studies in the literature at $\mu=0$, we have been able to construct the combinations of real-time diagrams leading to retarded correlators and to closed diagrams contributing to the free energy.

We have applied this formalism to the case of a pion gas, relevant for relativistic heavy-ion collisions between thermal and chemical freeze-out with nonzero pion number chemical potential $\mu_{\pi}(T)$. Our description is consistent if the $T$ dependence of $\mu_{\pi}$ encodes the time evolution of the plasma between those phases. The relevant diagrammatic scheme for temperatures below chiral restoration is chiral perturbation theory. In this framework, we have calculated the leading corrections to the ideal gas coming from chiral interactions. To leading order $\mathcal{O}\left(T^{6}\right)$ the corrections to the pressure can be expressed in terms of tadpole diagrams and are numerically rather small up to $T_{c}$. To next to leading order $\mathcal{O}\left(T^{8}\right)$, closed diagrams contributing to the free energy can be obtained from particle-changing processes, which signals the onset of the number conservation approximation breakup. Nevertheless, since $\mu_{\pi}$ is small for temperatures where those ChPT corrections become important, they can be reliably calculated and produce sizable deviations from the free gas. The results to that order agree reasonably well with a virial expansion analysis. Our results for thermodynamical observables show that both chiral interactions and $\mu_{\pi}$ tend to increase the pressure. The chiral restoration critical temperature decreases with increasing $\mu_{\pi}$, which would be of relevance only if chiral restoration takes place for lower temperatures than chemi- cal freeze-out. We have also calculated the isentropic $\mu_{\pi}(T)$ curve for different orders in the interactions. The corrections to the ideal gas show a significant reduction of the chemical freeze-out temperature, which is the expected effect of interactions, since they increase the probability of producing inelastic processes. The same effect had been observed previously in a free gas of pions and resonances.

Our approach allows one to derive thermal corrections to the pion self-energy at $\mu_{\pi} \neq 0$, from the leading-order ChPT diagrams, both for the real and imaginary parts of the retarded correlator. The imaginary part comes from a two-loop diagram, for which the use of RTF rules for the construction of the retarded function is crucial. After a detailed evaluation, our diagrammatic result is shown to coincide with the expected expressions from kinetic theory arguments. We have also discussed the role of higher-order pinching-pole contributions to self-energies, providing different arguments which support the fact that those corrections are subleading in our approach. The real part of the self-energy gives the thermal mass, which together with the condensate and the pion decay constant to the same order, satisfy the $\mu_{\pi} \neq 0$ extension of the Gell-Mann--OakesRenner relation. In addition, both the real and imaginary parts satisfy a Luscher-like relation in terms of the forward pion scattering amplitude. This relation allows one to calculate in the dilute regime the self-energy corrections for higher orders in the ChPT amplitudes, including unitarized expressions which have the physically expected energy behavior and reproduce the lightest resonance states. The results for the thermal mass show a clear decreasing both with $T$ and $\mu_{\pi}$ for $\mathcal{O}\left(p^{4}\right)$ and unitarized amplitudes. This suggests the interesting possibility of reaching Bose-Einstein condensation when the effective thermal mass approaches the chemical potential. This mechanism would require lower pion densities to reach BE condensation. We have discussed this possibility, which is a purely interacting effect, within the isentropic values and comparing the pion densities with those in the standard approach of considering the ideal gas BE limit $\mu \rightarrow m_{\pi}^{-}$with $m_{\pi}$ the vacuum mass. Our mass-dropping BE curve is not far, but still above the isentropic ones for reasonable values of chemical freeze-out. Finally, using also the scattering amplitudes, we have evaluated the corrections to the mean collision time at $\mu_{\pi} \neq 0$. The mean time decreases with $T$ and $\mu_{\pi}$ for all orders in the interaction, which implies a sizable reduction, compared to the $\mu_{\pi}=0$ case, of the thermal freeze-out temperature, estimated as that where $\tau$ equals the typical plasma lifetime.

Summarizing, the diagrammatic field-theory scheme developed in the present work provides, in our opinion, useful results regarding the chemically nonequilibrated phase of the meson gas resulting from a relativistic heavy-ion collision. In future work we plan to generalize the analysis presented here to include also the strange sector (kaons and eta) as well as to extend previous studies 
of transport coefficients by including pion chemical potentials along the lines presented here.

\section{ACKNOWLEDGMENTS}

We acknowledge financial support from the Spanish research Projects No. FPA2007-29115-E, No. PR341856-BSCH, No. CCG07-UCM/ESP-2628, No. FPA200800592, No. FIS2008-01323, and from the FPI programme (No. BES-2005-6726).

\section{APPENDIX A: HOLOMORPHIC PATH INTEGRALS}

We review here some of the key aspects of the holomorphic path-integral representation which are used in the main text. We will follow the discussion in [23], to which we refer for more details.

We consider the space $\mathcal{S}$ of complex analytic functions of one complex variable and define the following scalar product:

$$
\langle f \mid g\rangle \equiv \int \frac{d \bar{z} d z}{2 \pi i} e^{-\bar{z} z \bar{f}(z)} g(z),
$$

where the bar denotes complex conjugation ( $z$ and $\bar{z}$ are treated as independent variables), and the notation for the measure means

$$
\int \frac{d \bar{z} d z}{2 \pi i} \equiv \int_{-\infty}^{\infty} \frac{d x d y}{\pi}
$$

with $z \equiv x+i y$. We also define the states $\langle z|$ in the dual space $\mathcal{S}^{*}$ such that $\langle z \mid f\rangle \equiv f(z)$, with $|f\rangle \in \mathcal{S}$. Then, the set $\left\{f_{n}\right\}_{0}^{\infty}$, with

$$
f_{n}(z) \equiv \frac{z^{n}}{\sqrt{n !}}
$$

constitutes an orthonormal basis for $\mathcal{S}$ with the inner product (A1). This implies, in particular,

$$
\int \frac{d z^{\prime} d \bar{z}^{\prime}}{2 \pi i} e^{-z^{\prime} \bar{z}^{\prime}} e^{z^{\prime} z} f\left(z^{\prime}\right)=f(z)
$$

We can also calculate the scalar product:

$$
\left\langle z \mid \bar{z}^{\prime}\right\rangle=\sum_{n=0}^{\infty} f_{n}(z) \overline{f_{n}\left(z^{\prime}\right)}=\sum_{n=0}^{\infty} \frac{1}{n !}\left(z \bar{z}^{\prime}\right)^{n}=e^{z z^{\prime}},
$$

where we will denote the dual of $\langle z|$ by $|\bar{z}\rangle$. Now, from the definition (A1), the identity operator can be written as

$$
\hat{1}=\int \frac{d \bar{z} d z}{2 \pi i} e^{-\bar{z} z}|\bar{z}\rangle\langle z| .
$$

Since the functions (A3) constitute an orthonormal basis, we can calculate the trace of an operator as follows:

$$
\operatorname{Tr}\{\cdot\}=\sum_{n=0}^{\infty}\left\langle f_{n}|\cdot| f_{n}\right\rangle=\int \frac{d \bar{z} d z}{2 \pi i} e^{-\bar{z} z\langle z|\cdot| \bar{z}\rangle .}
$$

The prescription (6) defines a representation of the creation and annihilation operators on $\mathcal{S}$. Therefore,

$$
\begin{aligned}
\left\langle z\left|\hat{a}^{\dagger}\right| \bar{z}^{\prime}\right\rangle & =z\left\langle z \mid \bar{z}^{\prime}\right\rangle=z e^{z \bar{z}^{\prime}}, \\
\left\langle z|\hat{a}| \bar{z}^{\prime}\right\rangle & =\frac{\partial}{\partial z}\left\langle z \mid \bar{z}^{\prime}\right\rangle=\bar{z}^{\prime} e^{z \bar{z}^{\prime}} .
\end{aligned}
$$

For the purpose of obtaining a path integral, we need to know how to calculate the matrix elements (kernels) of the kind $\mathcal{O}\left(z, \bar{z}^{\prime}\right) \equiv\left\langle z\left|\hat{O}\left(\hat{a}^{\dagger}, \hat{a}\right)\right| \bar{z}^{\prime}\right\rangle$, where $\hat{O}$ is an operator expressed in terms of creation and annihilation operators. If the operator is expressed in normal-order form (which we denote by the subscript $\mathrm{N}$ ) i.e., arranging creation operators to the left and annihilation to the right, the kernels can be written in a particularly useful way, from (A8), as

$$
\left\langle z\left|\hat{O}_{\mathrm{N}}\right| \bar{z}^{\prime}\right\rangle=O_{\mathrm{N}}(z, \partial / \partial z) e^{z \bar{z}^{\prime}}=O_{\mathrm{N}}\left(z, \bar{z}^{\prime}\right) e^{z \bar{z}^{\prime}} .
$$

In particular, we will need the kernel corresponding to "time evolution":

$$
\mathcal{U}\left(z, \bar{z}^{\prime} ; t_{f}-t_{i}\right) \equiv\left\langle z\left|e^{-i\left(t_{f}-t_{i}\right) \hat{H}_{\mathrm{N}}}\right| \bar{z}^{\prime}\right\rangle,
$$

with $\hat{H}_{\mathrm{N}}$ the normal-ordered Hamiltonian of the system.

For that purpose, as customary, we divide the interval $t_{f}-t_{i}$ into $n$ subintervals of infinitesimal length $\varepsilon$ and we will take the $n \rightarrow \infty$ limit in the end. Having in mind the application to thermal field theory, we will take complex times $t \in C$ where $C$ is the contour starting at $t_{i}$ and ending at $t_{f}=t_{i}-i \beta$ as shown in Fig. 1.

Now, from (A9), for an infinitesimal time interval:

$$
\mathcal{U}\left(z_{1}, \bar{z}_{2} ; \varepsilon\right) \simeq e^{-i \varepsilon H_{\mathrm{N}}\left(z_{1}, \bar{z}_{2}\right)} e^{z_{1} \bar{z}_{2}},
$$

so that, inserting the identity operator (A6) $n-1$ times in (A10) one gets

$$
\begin{aligned}
& \mathcal{U}\left(z, \bar{z}^{\prime} ; t_{f}-t_{i}\right) \\
& =\int \prod_{k=1}^{n-1} \frac{d z_{k} d \bar{z}_{k}}{2 \pi i} \exp \left\{z_{1} \bar{z}^{\prime}+\sum_{k=1}^{n-1}\left[\left(z_{k+1}-z_{k}\right) \bar{z}_{k}\right.\right. \\
& \left.\left.\quad+\varepsilon \hat{H}_{\mathrm{N}}\left(z_{k+1}, \bar{z}_{k}\right)\right]\right\},
\end{aligned}
$$

with $z_{n}=z$.

For the Hamiltonian (3) one has $\hat{H}=\hat{H}_{\mathrm{N}}+\omega / 2$ and the previous integral can be explicitly calculated by using the standard formula [23]:

$$
\int \prod_{k=1}^{n} \frac{d z_{k} d \bar{z}_{k}}{2 \pi i} e^{-\bar{z} A z+\bar{u} z+u \bar{z}}=(\operatorname{det} A)^{-1} e^{\bar{u} A^{-1} u},
$$

which, taking the $n \rightarrow \infty$ limit, yields

$$
\mathcal{U}_{0}\left(z, \bar{z}^{\prime} ; t_{f}-t_{i}\right)=\exp \left(z \bar{z}^{\prime} e^{-i \omega\left(t_{f}-t_{i}\right)}+\Sigma[j]\right),
$$

where the subscript " 0 " distinguishes the particular case of the Hamiltonian (3) and 


$$
\begin{aligned}
\Sigma[j]= & i \int_{C} d t\left[z \frac{e^{-i \omega\left(t_{f}-t\right)}}{\sqrt{2 \omega}} j(t)+\bar{z}^{\prime} \frac{e^{i \omega\left(t_{i}-t\right)}}{\sqrt{2 \omega}} j(t)\right] \\
& -\int_{C} d t d t^{\prime} j(t) \theta\left(t-t^{\prime}\right) \frac{e^{-i \omega\left(t-t^{\prime}\right)}}{2 \omega} j\left(t^{\prime}\right) .
\end{aligned}
$$

\section{APPENDIX B: FREE THERMAL PROPAGATORS AND PARTITION FUNCTION AT $\boldsymbol{\mu} \neq 0$}

In this Appendix we review some important aspects regarding the canonical description of the free theory and the different representations for free propagators in thermal field theory at nonzero chemical potential, paying special attention to the differences between the case of particle number chemical potential and that of exactly conserved charges such as the electric charge for complex scalar fields.

Let us consider first the case of a free neutral scalar field $\phi(x)$. In that case, one can evaluate the partition function and the propagator (two-point function) directly in the complete set $\left|N_{1}, N_{2}, \ldots\right\rangle$, corresponding to eigenstates of the Hamiltonian operator with $N_{1}$ particles in state $1, N_{2}$ particles in state 2 , and so on:

$$
\begin{aligned}
\left\langle N_{1}, N_{2}, \ldots \mid N_{1}^{\prime}, N_{2}^{\prime}, \ldots\right\rangle= & \delta_{N_{1} N_{1}^{\prime}} \cdot \delta_{N_{2} N_{2}^{\prime}} \cdot \ldots, \\
\hat{H}_{0}\left|N_{1}, N_{2}, \ldots\right\rangle= & \left(N_{1} E_{1}+\cdots+\sum_{i=1}^{\infty} \frac{E_{i}}{2}\right) \\
& \times\left|N_{1}, N_{2}, \ldots\right\rangle, \\
\hat{N}\left|N_{1}, N_{2}, \ldots\right\rangle= & \left(N_{1}+N_{2}+\cdots\right)\left|N_{1}, N_{2}, \ldots\right\rangle,
\end{aligned}
$$

with $\sum N_{i}=N$. For noninteracting bosons of mass $m$, $E_{i} \equiv \sqrt{m^{2}+\left|\vec{p}_{i}\right|^{2}}$, and the (infinite) term $\sum_{i} E_{i} / 2$ is the vacuum energy. As customary, we consider first the system in a finite volume $V=L^{3}$, which we will later take to infinity, so that spatial momenta are discretized as $\left|p_{i}\right|=$ $\frac{\pi n_{i}}{L}$ with integers $n_{i}$ and energy levels are labeled by $\vec{n} \equiv$ $\left(n_{x}, n_{y}, n_{z}\right)$. The free partition function reads then

$$
\tilde{Z}_{\beta}^{0}=\prod_{\vec{n}} \sum_{N=0}^{\infty} e^{-\beta N\left(E_{\vec{n}}-\mu\right)} e^{-\beta E_{\vec{n}} / 2}=\prod_{\vec{n}} \frac{e^{-\beta E_{\vec{n}} / 2}}{1-e^{-\beta\left(E_{\vec{n}}-\mu\right)}},
$$

where the condition $\mu<E_{\vec{n}}$ must be satisfied for all $\vec{n}$. Thus, in the $V \rightarrow \infty$ limit:

$$
\log \tilde{Z}_{\beta}^{0}=-V \int \frac{d^{3} \vec{p}}{(2 \pi)^{3}}\left[\frac{\beta E_{p}}{2}+\log \left(1-e^{-\beta\left(E_{p}-\mu\right)}\right)\right],
$$

where $E_{p}^{2}=|\vec{p}|^{2}+m^{2}$. Therefore, in the following we must restrict to a chemical potential $\mu \leq m$ (below the Bose-Einstein condensation limit) to ensure the convergence of the previous expressions.
In order to obtain the free particle propagator in the canonical formalism, defined as the two-point function:

$$
\begin{aligned}
\tilde{G}(x) & \equiv\langle\hat{T} \hat{\phi}(x) \hat{\phi}(0)\rangle_{\beta, \mu} \\
& \equiv \tilde{Z}_{\beta}^{-1} \operatorname{Tr}\left\{e^{-\beta(\hat{H}-\mu \hat{N})} \hat{T} \hat{\phi}(x) \hat{\phi}(0)\right\},
\end{aligned}
$$

where $\hat{T}$ is the time-ordering operator, we expand the field as customary in terms of creation and annihilation operators:

$$
\hat{\phi}(\vec{x})=\frac{1}{V} \sum_{n} \frac{1}{\sqrt{2 E_{\vec{n}}}}\left(\hat{a}_{\vec{n}} e^{i 2 \pi \vec{n} \cdot \vec{x} / L}+\hat{a}_{\vec{n}}^{\dagger} e^{-i 2 \pi \vec{n} \cdot \vec{x} / L}\right),
$$

with commutation relation

$$
\left[\hat{a}_{\vec{n}}, \hat{a}_{\vec{n}^{\prime}}^{\dagger}\right]=V \delta_{\vec{n}, \vec{n}^{\prime}} .
$$

The free Hamiltonian and the number operator are given in terms of creation and annihilation operators as

$$
\hat{H}_{0}=\sum_{\vec{n}} \frac{1}{V} E_{\vec{n}}\left(\hat{a}_{\vec{n}}^{\dagger} \hat{a}_{\vec{n}}+\frac{1}{2} V\right), \quad \hat{N}=\sum_{\vec{n}} \frac{1}{V} \hat{a}_{\vec{n}}^{\dagger} \hat{a}_{\vec{n}} .
$$

Now, the real time evolution of the field is given by $\hat{\phi}(t, \vec{x}) \equiv e^{i \hat{H} t} \hat{\phi}(\vec{x}) e^{-i \hat{H} t}$ with $t \in \mathbb{R}$. We will calculate the trace in (B3) using

$$
\left\langle\frac{1}{V} \hat{a}_{\vec{n}}^{\dagger} \hat{a}_{\vec{n}}\right\rangle_{\beta, \mu}=\frac{1}{e^{\beta\left(E_{\vec{n}}-\mu\right)}-1} \equiv n\left(E_{\vec{n}}-\mu\right),
$$

so that we get for the free propagator, after taking the $V \rightarrow$ $\infty$ limit:

$$
\tilde{G}(x)=\theta(t) \tilde{G}^{>}(x)+\theta(-t) \tilde{G}^{<}(x),
$$

with

$$
\begin{aligned}
& \tilde{G}^{>(<)}(x)=\int \frac{d^{3} \vec{p}}{(2 \pi)^{3}} e^{i \vec{p} \cdot \vec{x}} \tilde{G}^{>(<)}(t, p), \\
& \tilde{G}^{>}(t, p)= \frac{1}{2 E_{p}}\left[e^{-i E_{p} t}\left(1+n\left(E_{p}-\mu\right)\right)\right. \\
&\left.+e^{i E_{p} t} n\left(E_{p}-\mu\right)\right], \\
& \tilde{G}^{<}(t, p)= \frac{1}{2 E_{p}}\left[e^{i E_{p} t}\left(1+n\left(E_{p}-\mu\right)\right)\right. \\
&\left.+e^{-i E_{p} t} n\left(E_{p}-\mu\right)\right] .
\end{aligned}
$$

Note that the above propagators are obtained from the $\mu=0$ ones by the following replacement in the distribution function:

$$
n(x) \rightarrow \tilde{n}_{p}(x) \equiv \frac{1}{e^{\tilde{\beta}_{p} x}-1},
$$

with 


$$
\tilde{\beta}_{p} \equiv \beta\left(1-\frac{\mu}{E_{p}}\right)
$$

Therefore, we have for instance $\tilde{n}_{p}\left(E_{p}\right)=n\left(E_{p}-\mu\right)$ and the $\tilde{n}_{p}$ function satisfie:

$$
1+\tilde{n}_{p}(x)+\tilde{n}_{p}(-x)=0 .
$$

Thus, the free propagator satisfies the following KMSlike periodicity condition in the mixed representation:

$$
\tilde{G}^{>}(t, p)=\tilde{G}^{<}\left(t+i \tilde{\beta}_{p}, p\right)
$$

and in Fourier space we can write a spectral representation:

$$
\begin{aligned}
\tilde{G}^{>}\left(p_{0}, p\right) & =\left[1+\tilde{n}_{p}\left(p_{0}\right)\right] \rho\left(p_{0}, p\right) \tilde{G}^{<}\left(p_{0}, p\right) \\
& =e^{-\tilde{\beta}_{p} p_{0}} \tilde{G}^{>}\left(p_{0}, p\right)=\tilde{n}_{p}\left(p_{0}\right) \rho\left(p_{0}, p\right),
\end{aligned}
$$

where

$$
\rho\left(p_{0}, p\right)=2 \pi \operatorname{sgn}\left(p_{0}\right) \delta\left[\left(p_{0}\right)^{2}-E_{p}^{2}\right]
$$

is the free spectral function, which is independent of temperature and chemical potential.

Now, using

$$
\theta(t)=i \int_{-\infty}^{\infty} \frac{d k_{0}}{2 \pi} \frac{e^{-i k_{0} t}}{k_{0}+i \epsilon},
$$

with $\epsilon \rightarrow 0^{+}$, we can write for the propagator in (B8) in momentum space:

$$
\tilde{G}\left(p_{0}, p\right)=\frac{i}{p_{0}^{2}-E_{p}^{2}+i \epsilon}+2 \pi \delta\left(p_{0}^{2}-E_{p}^{2}\right) n\left(\left|p_{0}\right|-\mu\right) .
$$

Note that we have used $\tilde{n}_{p}\left(E_{p}\right) \delta\left(p_{0}^{2}-E_{p}^{2}\right)=$ $\tilde{n}_{p}\left(\left|p_{0}\right|\right) \delta\left(p_{0}^{2}-E_{p}^{2}\right)=n\left(\left|p_{0}\right|-\mu\right) \delta\left(p_{0}^{2}-E_{p}^{2}\right)$ and we have chosen the " $\left|p_{0}\right|$ prescription" which, as explained in the main text, guarantees the decoupling of the imaginary-leg contribution to real-time Green functions.

The free propagators in (B10) and (B11) can be extended to imaginary times $t=-i \tau$ corresponding time differences along the imaginary-time leg $C_{4}$ in Fig. 1. Thus, we define $\tilde{\Delta}_{T}(\tau, p)=\tilde{G}^{>}(-i \tau, p)$ for $\tau \geq 0$ and $\tilde{\Delta}_{T}(\tau, p)=\tilde{G}^{<}(-i \tau, p)$ for $\tau \leq 0$. Now, if we try to construct a Matsubara frequency representation in this case, we have, from the mixed representation (B10) and using (B12) and (B13):

$$
\begin{aligned}
\tilde{\Delta}_{T}(\tau \geq 0, p) & =\frac{1}{2 \pi i} \oint_{C_{1} \cup C_{2}} \frac{e^{z \tau}}{e^{\tilde{\beta}_{p} z}-1} \frac{1}{z^{2}-E_{p}^{2}} \\
& \stackrel{0 \leq ⿻ \tilde{\beta}_{p}}{=} \frac{1}{\tilde{\beta}_{p}} \sum_{n} \frac{e^{i\left(\tilde{\omega}_{n}\right)}}{\tilde{\omega}_{n}^{2}+E_{p}^{2}},
\end{aligned}
$$

where the $C_{1,2}$ contours are shown in Fig. 10, the black dots on the imaginary axis being the "modified" Matsubara frequencies $\tilde{\omega}_{n}=2 \pi n / \tilde{\beta}_{p}$. A very important point here is

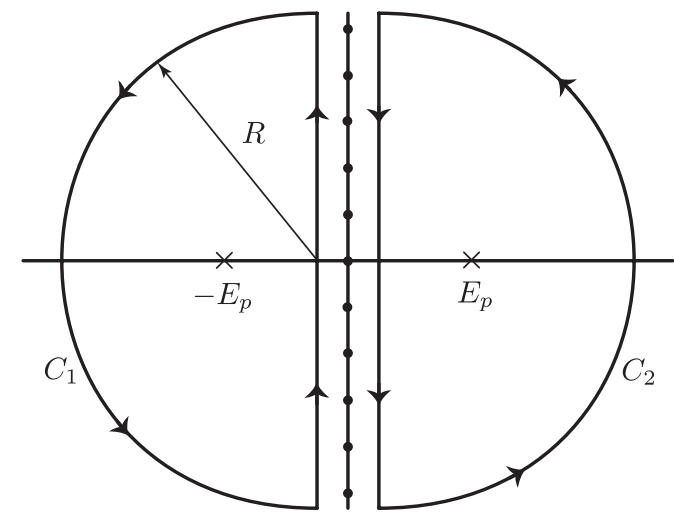

FIG. 10. Contours used to derive the Matsubara representation of the free imaginary-time propagators. The black dots on the imaginary axis denote the Matsubara frequencies and $R \rightarrow \infty$.

that the last step in the above equation is only valid for $\tau \in$ $\left[0, \tilde{\beta}_{p}\right]$, otherwise the integrals along the circular arcs with $R \rightarrow \infty$ do not vanish. Thus, the Matsubara Fourier representation is only valid in that interval, which is smaller than $[0, \beta]$.

Carrying out the same procedure with $\tilde{\Delta}_{T}(\tau \leq 0, p)$ using (B11) leads to the same modified Matsubara representation for $\tau \in\left[-\tilde{\beta}_{p}, 0\right]$. In fact, we see that the KMSlike condition (B15) translates into the imaginary-time propagator as

$$
\tilde{\Delta}_{T}\left(\tau+\tilde{\beta}_{p}, p\right)=\tilde{\Delta}_{T}(\tau, p)
$$

so that this propagator does not satisfy the usual equilibrium KMS condition $\tilde{\Delta}_{T}(\tau+\beta, p)=\tilde{\Delta}_{T}(\tau, p)$.

At this point, it is instructive to compare the above free propagators for chemical nonequilibrium with those obtained when an exact conserved charge is present. As we are going to see, there are crucial differences between the two cases. For definiteness, we consider the electric charge for the case of a complex scalar field and denote the corresponding chemical potential by $\mu_{Q}$. In that case, the counterparts of (B10) and (B11) for the free propagator $G_{Q}(x)=\left\langle\hat{T} \hat{\phi}^{\dagger}(x) \hat{\phi}(0)\right\rangle$ are [17]

$$
\begin{aligned}
G_{Q}^{>}(t, p)= & \frac{1}{2 E_{p}}\left[e^{-i E_{p} t}\left(1+n\left(E_{p}-\mu_{Q}\right)\right)\right. \\
& \left.+e^{i E_{p} t} n\left(E_{p}+\mu_{Q}\right)\right], \\
G_{Q}^{<}(t, p)= & \frac{1}{2 E_{p}}\left[e^{i E_{p} t}\left(1+n\left(E_{p}+\mu_{Q}\right)\right)\right. \\
& \left.+e^{-i E_{p} t} n\left(E_{p}-\mu_{Q}\right)\right] .
\end{aligned}
$$

Note that, unlike our previous case in (B10) and (B11), the chemical potential enters now with opposite sign for the positive and negative frequencies, which comes from the opposite charge of particles and antiparticles, necessary to maintain the chemical equilibrium imposed by charge 
conservation. Because of this sign difference, the above propagator satisfies now the following condition:

$$
G_{Q}^{>}(t, p)=e^{-\beta \mu_{Q}} G_{Q}^{<}(t+i \beta, p),
$$

i.e., in this case the KMS symmetry realizes simply as a modification of the $\mu_{Q}=0 \mathrm{KMS}$ boundary condition by a constant $\mu_{Q}$-dependent factor, which is a result completely different from the previous case, cf., Eq. (B15), where the loss of KMS involves the $p$-dependent $\tilde{\beta}_{p}$, which cannot be rewritten as a multiplicative factor:

$$
\begin{aligned}
\tilde{G}^{>}(t, p)= & \frac{1}{2 E_{p}}\left[e^{-\beta \mu_{\pi}} e^{-i E_{p}(t+i \beta)} n\left(E_{p}-\mu_{\pi}\right)\right. \\
& \left.+e^{\beta \mu_{\pi}} e^{i E_{p}(t+i \beta)}\left(1+n\left(E_{p}-\mu_{\pi}\right)\right)\right] \\
\neq & e^{-\beta \mu_{\pi}} \tilde{G}^{<}(t+i \beta, p) .
\end{aligned}
$$

Another way to arrive to the same conclusion is to derive directly the periodicity relation from the thermal averages. In the charged scalar case, it is crucial to use that fact that the field is a charge eigenstate, i.e., $[\hat{Q}, \hat{\phi}]=-\hat{\phi}$, $\left[\hat{Q}, \hat{\phi}^{\dagger}\right]=\hat{\phi}^{\dagger}[17]$. This, together with charge conservation $[\hat{Q}, \hat{H}]=0$ leads to

$$
\begin{aligned}
& \operatorname{Tr}\left[\hat{\phi}^{\dagger}(t-i \beta) \hat{\phi}(0) e^{-\beta\left(\hat{H}-\mu_{Q} \hat{Q}\right)}\right] \\
& \quad=e^{-\beta \mu_{Q}} \operatorname{Tr}\left[\hat{\phi}(t) \hat{\phi}^{\dagger}(0) e^{-\beta\left(\hat{H}-\mu_{Q} \hat{Q}\right)}\right] .
\end{aligned}
$$

However, in the case of a real field and the number operator, even though $[\hat{N}, \hat{H}]=0$ in the free case, $[\hat{N}, \hat{\phi}] \neq \pm \hat{\phi}$ which prevents the previous relation from holding.

Defining now the imaginary-time propagators as above, we get the same factor in the $\mu_{Q}$ case:

$$
\Delta_{T}^{Q}(\tau+\beta, p)=e^{-\beta \mu_{Q} \Delta_{T}^{Q}}(\tau, p) .
$$

In fact, it is not difficult to see that in the $\mu_{Q}$ case, this simple form of KMS symmetry still allows for a welldefined Matsubara IT frequency representation:

$$
\begin{aligned}
& \tilde{\Delta}_{T}^{Q}(\tau \geq 0, p)=\frac{1}{2 \pi i} \oint_{C_{1}^{\prime} \cup C_{2}^{\prime}} \frac{e^{z \tau}}{e^{\beta\left(z+\mu_{Q}\right)}-1} \frac{1}{z^{2}-E_{p}^{2}} \\
& \stackrel{0 \leq \tau \leq \beta}{=} \frac{1}{\beta} \sum_{n} \frac{e^{i\left(\omega_{n}+i \mu_{Q}\right)}}{\left(\omega_{n}+i \mu_{Q}\right)^{2}+E_{p}^{2}},
\end{aligned}
$$

where $C_{12}^{\prime}$ correspond to the contours in Fig. 10 but with the vertical line displaced to $z=-\mu_{Q}\left(\mu_{Q}<m\right)$ and the dots in that line being now the standard Matsubara frequencies $\omega_{n}=2 \pi n / \beta$. Therefore, in this case the ordi- nary IT formalism is recovered for $\tau \in[-\beta, \beta]$ simply by changing in the Feynman rules $\omega_{n} \rightarrow \omega_{n}+i \mu_{Q}$.

Most of the results shown in the main text can be written in terms of the above thermal propagators evaluated at the origin in position space and functions related to them. From (B9)-(B11) we have (for $\mu \leq m$ ) at $\tau=t=\vec{x}=0$ :

$$
\begin{aligned}
\tilde{G}^{>}(0) & =\tilde{G}^{<}(0)=\tilde{\Delta}_{T}(0)=\tilde{G}(0) \\
& =[\tilde{G}(0)]^{T=\mu=0}+\tilde{g}_{1}(m, T, \mu),
\end{aligned}
$$

where the $T=\mu=0$ contribution is ultraviolet divergent. In dimensional regularization it is given by

$$
[\tilde{G}(0)]^{T=\mu=0}=\int \frac{d^{D-1} p}{(2 \pi)^{D-1}} \frac{1}{2 E_{p}}=\frac{\Gamma\left[1-\frac{D}{2}\right] m^{D-2}}{(4 \pi)^{D / 2}},
$$

while the $T, \mu$-dependent contribution $\tilde{g}_{1}$ is finite. We are following the same notation as in [31] so that $\tilde{g}_{1}$ is the $\mu \neq$ 0 extension of their function $g_{1}(T)$, to which it reduces for $\mu=0$. We have

$$
\tilde{g}_{1}(m, T, \mu)=\frac{1}{2 \pi^{2}} \int_{0}^{\infty} d p \frac{p^{2}}{E_{p}} \frac{1}{e^{\beta\left(E_{p}-\mu\right)}-1} .
$$

Note that in dimensional regularization one has, as in the $\mu=0$ case,

$$
\begin{aligned}
{\left.\left[\partial_{\tau}^{2}-\nabla^{2}\right] \tilde{\Delta}_{T}(\tau, \vec{x})\right|_{\tau=\vec{x}=0} } & =m^{2} \tilde{\Delta}_{T}(0), \\
\left.\square \tilde{G}(t, \vec{x})\right|_{t=\vec{x}=0} & =-m^{2} \tilde{G}(0),
\end{aligned}
$$

and $\partial_{\mu} \tilde{G}(0)=\partial_{\mu} \Delta_{T}(0)=0$.

Let us also define, following again the notation in [31]:

$$
\tilde{g}_{0}(m, T, \mu)=-\frac{T}{\pi^{2}} \int_{0}^{\infty} d p p^{2} \log \left[1-e^{-\beta\left(E_{p}-\mu\right)}\right]
$$

so that, taking into account that $\partial E_{p} / \partial m^{2}=1 /\left(2 E_{p}\right)$, we can write the free partition function (B32) separating its divergent contribution in dimensional regularization as

$$
\log \tilde{Z}_{\beta}^{0}=\frac{\beta V}{2}\left[\frac{\Gamma\left[-\frac{D}{2}\right] m^{D}}{(4 \pi)^{D / 2}}+\tilde{g}_{0}(m, T, \mu)\right] .
$$

Finally, note that the functions $\tilde{g}_{0}$ and $\tilde{g}_{1}$ satisfy a similar relation as in [31]:

$$
\tilde{g}_{1}(m, T, \mu)=-\frac{\partial}{\partial m^{2}} g_{0}(m, T, \mu) .
$$

[1] M. Kataja and P. V. Ruuskanen, Phys. Lett. B 243, 181 (1990).
[2] H. Bebie, P. Gerber, J. L. Goity, and H. Leutwyler, Nucl. Phys. B378, 95 (1992). 
[3] C. M. Hung and E. V. Shuryak, Phys. Rev. C 57, 1891 (1998).

[4] P. Braun-Munzinger, K. Redlich, and J. Stachel, in Quark Gluon Plasma 3, edited by R. C. Hwa and Xin-Nian Wang (World Scientific Publishing, Singapore, 2004), pp. 491599.

[5] P.F. Kolb and R. Rapp, Phys. Rev. C 67, 044903 (2003).

[6] G. Torrieri, S. Jeon, and J. Rafelski, Phys. Rev. C 74, 024901 (2006).

[7] J. Letessier and J. Rafelski, Eur. Phys. J. A 35, 221 (2008).

[8] C. Song and V. Koch, Phys. Rev. C 55, 3026 (1997).

[9] J.L. Goity and H. Leutwyler, Phys. Lett. B 228, 517 (1989).

[10] A. Schenk, Phys. Rev. D 47, 5138 (1993).

[11] J. L. Goity, Phys. Lett. B 319, 401 (1993).

[12] M. Prakash, M. Prakash, R. Venugopalan, and G. Welke, Phys. Rep. 227, 321 (1993).

[13] A. Dobado and F. J. Llanes-Estrada, Phys. Rev. D 69, 116004 (2004); A. Dobado, F. J. Llanes-Estrada, and J. M. Torres-Rincon, Phys. Rev. D 79, 014002 (2009).

[14] A. Dobado and J. R. Pelaez, Phys. Rev. D 59, 034004 (1998).

[15] R. Baier, M. Dirks, and K. Redlich, Phys. Rev. D 55, 4344 (1997).

[16] K. c. Chou, Z. b. Su, B. 1. Hao, and L. Yu, Phys. Rep. 118, 1 (1985).

[17] N. P. Landsman and C. G. van Weert, Phys. Rep. 145, 141 (1987).

[18] C. Adler et al. (STAR Collaboration), Phys. Rev. Lett. 87, 082301 (2001).

[19] V.L. Eletsky, J. I. Kapusta, and R. Venugopalan, Phys. Rev. D 48, 4398 (1993).

[20] D. T. Son and M. A. Stephanov, Phys. Rev. Lett. 86, 592 (2001); M. Loewe and C. Villavicencio, Phys. Rev. D 67, 074034 (2003).

[21] J.I. Kapusta and C. Gale, Finite-Temperature Field Theory: Principles and Applications (Cambridge University Press, Cambridge, England, 2006).

[22] C. Itzykson and J.B. Zuber, Quantum Field Theory (McGraw-Hill, New York, 1980).

[23] J. Zinn-Justin, Quantum Field Theory and Critical Phenomena (Oxford University Press, New York, 2002), 4th ed..
[24] M. Le Bellac, Thermal Field Theory (Cambridge University Press, Cambridge, England, 1996).

[25] T. Altherr, Phys. Lett. B 341, 325 (1995).

[26] A. Niegawa, Phys. Rev. D 40, 1199 (1989).

[27] F. Gelis, Phys. Lett. B 455, 205 (1999).

[28] R. Kobes, Phys. Rev. D 42, 562 (1990); 43, 1269 (1991).

[29] Y. Fujimoto, H. Matsumoto, H. Umezawa, and I. Ojima, Phys. Rev. D 30, 1400 (1984); 31, 1527(E) (1985); H. Matsumoto, Y. Nakano, and H. Umezawa, Phys. Rev. D 31, 1495 (1985).

[30] J. Gasser and H. Leutwyler, Ann. Phys. (N.Y.) 158, 142 (1984).

[31] P. Gerber and H. Leutwyler, Nucl. Phys. B321, 387 (1989).

[32] S. Weinberg, Physica A (Amsterdam) 96, 327 (1979).

[33] D. Fernandez-Fraile and A. Gomez Nicola, Phys. Rev. Lett. 102, 121601 (2009); Eur. Phys. J. C 62, 37, (2009).

[34] J. Gasser and H. Leutwyler, Phys. Lett. B 184, 83 (1987).

[35] M. Gell-Mann, R. J. Oakes, and B. Renner, Phys. Rev. 175, 2195 (1968)

[36] D. Toublan, Phys. Rev. D 56, 5629 (1997).

[37] M. Luscher, Commun. Math. Phys. 104, 177 (1986).

[38] T. N. Truong, Phys. Rev. Lett. 61, 2526 (1988); 67, 2260 (1991); A. Dobado, M. J. Herrero, and T. N. Truong, Phys. Lett. B 235, 134 (1990); A. Dobado and J. R. Peláez, Phys. Rev. D 47, 4883 (1993); 56, 3057 (1997); A. Gómez Nicola and J. R. Peláez, Phys. Rev. D 65, 054009 (2002).

[39] A. Dobado, A. Gomez Nicola, F. J. Llanes-Estrada, and J. R. Pelaez, Phys. Rev. C 66, 055201 (2002).

[40] A. Ayala, P. Amore, and A. Aranda, Phys. Rev. C 66, 045205 (2002); A. Ayala and J. Magnin, Phys. Rev. C 68, 014902 (2003).

[41] R. F. Sawyer, Phys. Rev. Lett. 29, 382 (1972); D. B. Kaplan and A. E. Nelson, Phys. Lett. B 175, 57 (1986).

[42] J. Zimanyi, G. I. Fai, and B. Jakobsson, Phys. Rev. Lett. 43, 1705 (1979); C. Greiner, C. Gong, and B. Muller, Phys. Lett. B 316, 226 (1993); R. Lednicky, V. Lyuboshitz, K. Mikhailov, Yu. Sinyukov, A. Stavinsky, and B. Erazmus, Phys. Rev. C 61, 034901 (2000); V. V. Begun and M. I. Gorenstein, Phys. Lett. B 653, 190 (2007).

[43] D. Fernandez-Fraile and A. Gomez Nicola, Phys. Rev. D 73, 045025 (2006); Int. J. Mod. Phys. E 16, 3010 (2007); Eur. Phys. J. A 31, 848 (2007). 